\title{
Globalización, expansión de la frontera y desigualdad en Chile durante el auge salitrero (1880-1905)
}

\section{Globalization, frontier expansion and inequality in Chile during the Saltpeter Heyday (1880-1905)}

JAVIER E. RODRÍGUEZ WEBER

Universidad de la República, Uruguay

\begin{abstract}
RESUMEN
Según una abundante literatura de orientación neoclásica, la primera globalización condujo a un aumento de la desigualdad en América Latina como resultado del efecto señalado por el teorema Heckscher-Ohlin-Samuelson. Esta línea argumental ha estado sujeta a importantes cuestionamientos dados los supuestos simplificadores del modelo. Pero incluso desde una perspectiva neoclásica este análisis resulta insuficiente. A partir de estimaciones recientes de la distribución del ingreso en Chile para el período 1860-1930 mostramos que el cambio en la dotación de factores que supuso la extensión de la frontera en la década de 1880 condujo a resultados diferentes a los predichos por el modelo Heckscher-Ohlin. Así entre 1880 y 1905, aunque Chile profundiza su especialización en productos primarios para la exportación, la expansión al norte y sur del tradicional Valle Central hizo más abundante la tierra en relación al trabajo y propició una serie de movimientos demográficos internos que condujeron a una reducción de la desigualdad.
\end{abstract}

PALABRAS CLAVE: Desigualdad, Chile, Primera Globalización, Expansión de la frontera

Código JEL: 015, N96, F11, J61

\section{ABSTRACT}

The literature on Latin American inequality during the First Globalization inspired by the Heckscher-Ohlin-Samuelson model suggests that inequality rose because of specialization in natural resource-intensive goods. However, this line of research has been criticized because of the simplifications and assumptions of the model. In this paper we argue that even in a neoclassical framework it must be revised in the light of changes in relative factor endowments, such as the expansion of the frontier - a common process in that period. Using a set of new estimates of income inequality in Chile between 1880 and 1905, we show that the expansion of the frontier opened up a process of social change and labor force migrations which improved the income distribution.

KEYWORDS: Income distribution, Chile, First Globalization, Expansion of the frontier

JEL Codes: 015, N96, F11, J61 


\section{Introducción ${ }^{1}$}

$\mathrm{E}$ n los últimos años se ha desarrollado una amplia literatura de orientación neoclásica interesada en estudiar el impacto de la Primera Globalización en la distribución del ingreso en América Latina². Inspirada en la extensión de Stolper \& Samuelson del modelo de Heckscher-Ohlin (H-O-S-S), su argumento central es que como resultado de la mayor integración del continente al comercio mundial, y dada su especialización en bienes intensivos en recursos naturales durante ese período, la desigualdad debió aumentar.

Esta línea argumental ha estado sujeta a importantes cuestionamientos debido a los supuestos simplificadores del modelo, que desconoce los factores institucionales y tecnológicos que median entre la especialización productiva, la inserción internacional y la distribución del ingreso ${ }^{3}$. Enmarcados en esta literatura crítica, aquí sostendremos que incluso desde una perspectiva neoclásica el análisis centrado en el modelo de Heckscher-Ohlin resulta insuficiente. A partir de evidencia reciente de la distribución del ingreso en Chile para el período 1860-1930 mostramos que el cambio en la dotación de factores causado por la extensión de la frontera en la década de 1880 condujo a resultados diferentes a los predichos por el modelo H-O-S-S. Aunque entre 1880 y 1905 Chile profundiza su especialización exportadora en bienes intensivos en recursos naturales — fundamentalmente la minería del salitre-, la expansión al norte y sur del tradicional Valle Central hizo más abundante la tierra en relación al trabajo y propició una serie de movimientos demográficos internos que condujeron a una reducción de la desigualdad. De esta forma, el modelo H-OS-S resulta incapaz de explicar lo que ocurre con la distribución del ingreso en un marco de cambio en la dotación de factores como el que supone la expansión de la frontera, fenómeno habitual durante la primera globalización.

El trabajo continúa de la siguiente manera. En la sección 2 abordamos algunos aspectos teóricos que hacen a la relación entre la globalización, frontera y desigualdad. En la sección 3 describimos la evolución de las variables de población y crecimiento durante el período, y cuestionamos las estimaciones de desigualdad preexis-

[Fecha de recepción del original, 4 de marzo de 2010. Versión definitiva, 1 de septiembre de 2010]

1 Este trabajo se basa en el capítulo VII de mi tesis de Maestría en Historia económica. Muchas personas me ayudaron en el proceso de investigación que condujo a la misma. En especial quiero agradecer a Luis Bertola quien la orientó, y a Carolina Román, Cecilia Castelnovo, Reto Bertoni, Henry Willebald, Jorge Álvarez, Paola Azar, José Díaz, José Jofré y Vicente Neira. Agradezco también a los participantes de los Seminarios de Historia Económica de Barcelona y Montevideo y de la sesión N. ${ }^{\circ}$ VI del CLADHE 2, así como a la Comisión Sectorial de Investigación Científica de la Universidad de la República, bajo cuyos auspicios comenzó esta investigación. Finalmente, deseo expresar mi agradecimiento a los árbitros cuyos comentarios me permitieron mejorar este artículo.

Ver Williamson $(1999,2002,2009)$ y Prados $(2005,2007)$.

Robinson (2001); Álvarez, Bertola \& Porcile (2007); Bértola \& Rodríguez Weber (2009); Bertola et al. (2010). 
tentes. En la sección 4 explicamos brevemente la metodología utilizada en la construcción de nuestras estimaciones de desigualdad. En la sección 5 mostramos los resultados obtenidos respecto a la evolución de la desigualdad durante nuestro período. En la sección 6 analizamos los mecanismos por los cuales la incorporación de nuevos territorios impactaron en la distribución del ingreso. Finalmente la sección 7 cierra con las conclusiones.

\section{Globalización, frontera y desigualdad}

\subsection{Economía internacional, globalización y desigualdad}

Entre las fuerzas dinámicas que hacen al desenvolvimiento económico de América latina, la inserción internacional constituye uno de los factores tradicionalmente señalados. Durante nuestro período de estudio desataca la integración internacional de los mercados de bienes y factores, proceso conocido como "primera globalización". Ésta ha sido analizada en particular por O’Rourke \& Williamson, quienes se interesan fundamentalmente por la integración de los mercados. Así definida la globalización, su impacto en la distribución del ingreso ha sido analizado en particular por Williamson en diversos trabajos individuales y colectivos ${ }^{4}$.

El argumento es simple y potente. Basado en la extensión de Stolper \& Samuelson del modelo de Heckscher-Ohlin, se señala que la integración de los mercados mundiales de bienes conduce a la convergencia en los precios de los factores, lo que tiene efectos redistributivos ${ }^{5}$. Según el teorema Heckscher-Ohlin, cuando una economía entra en el juego de la globalización se especializa en producir bienes intensivos en el factor que posee en abundancia. Entonces se produce un aumento en la demanda del factor abundante, lo que conduce a un aumento de la retribución a dicho factor en relación al factor escaso. De allí los efectos redistributivos del comercio internacional.

En el caso de las economías de América latina, la globalización reforzó la especialización en bienes intensivos en recursos naturales como alimentos y minerales, lo que supuso un aumento en la retribución al factor tierra. Lo anterior habría conducido a una disminución de la relación salario/renta de la tierra, y dado que la propiedad de ésta se concentra en un pequeño y privilegiado sector, ello supone un aumento en la desigualdad. En regiones con abundancia del factor trabajo la globalización habría conducido por el contrario a un aumento de la relación salario/ renta, y por tanto a una mayor igualdad ${ }^{6}$.

Ver O'Rourke \& Williamson (1999); Williamson (1998, 1999, 2002, 2009); Lindert \& Williamson (2001); Bertola \& Williamson (2006). Este enfoque también ha sido desarrollado por Prados (2005, 2007).

Stolper \& Samuelson (1941); Samuelson (1948).

Lindert \& Williamson (2001), p. 13. 
El trabajo realizado por Williamson junto a diversos coautores ha sido enormemente influyente ${ }^{7}$. Sin embargo, el mismo no ha estado exento de críticas. Así, Harley señala que al centrarse en una dimensión de la globalización -la integración de los mercados- quedan fuera importantes aspectos del proceso, alguno de los cuales resultan particularmente relevantes para el estudio de la distribución del ingreso. En este sentido, reclama un enfoque más amplio, centrado en la expansión de la economía atlántica sobre la región periférica y la expansión de la frontera ${ }^{8}$.

\subsection{Cambios en la dotación de factores}

El teorema Heckscher-Ohlin ha sido la respuesta a la pregunta sobre los efectos de la globalización en la retribución a los factores. Vimos que con el comercio aumenta la retribución al factor abundante. Ahora nos preguntamos qué ocurre en el caso de que se modifique la dotación de factores debido al aumento de uno en relación a otro. En nuestro caso nos interesa el aumento en la disponibilidad del factor tierra, producto de una expansión de la frontera.

Una primera aproximación formal en el marco de la economía neoclásica se encuentra en el trabajo de Rybczynski quien analizó el efecto de la ampliación de un factor productivo sobre la producción y los términos de intercambio entre los bienes. Utilizando un diagrama de caja similar al que usaran Stolper \& Samuelson', Rybczynski muestra que en el marco de una economía cerrada con dos factores $(X, Y)$ que produce dos bienes $(K, L)$, cada uno intensivo en un factor ( $K$ intensivo en $X$; $\mathrm{L}$ intensivo en $\mathrm{Y}$ ), la ampliación de un factor productivo $(\mathrm{X})$ conducirá al aumento de la producción del bien intensivo en dicho factor $(\mathrm{K})$, y a una reducción de la producción del bien intensivo en el factor que se ha vuelto relativamente más escaso (L) (Rybczynski, 1955: § 5). Asimismo, los términos de intercambio entre ambos bienes se ven afectados, de forma que el precio del bien intensivo en el factor creciente ( $\mathrm{K}$ intensivo en $\mathrm{X}$ ) cae respecto al precio del bien intensivo en el factor relativamente más escaso ( $\mathrm{L}$ intensivo en $\mathrm{Y}$ ):

"the terms of trade of the X-intensive commodity have worsened as against the Y-intensive commodity, and proves the proposition that the terms of trade of the commodity using relatively much of the factor whose quantity has increased must deteriorate (...) Our conclusion is that an increase in the quantity of one factor will always lead to a

Greasley et al. (2007).

Harley (2007), pp. 240-241.

Stolper \& Samuelson (1941), Fig. 2, p. 69. 
worsening in the terms of trade, or the relative price, of the commodity using relatively much of that factor" ${ }^{10}$.

De forma que un corrimiento de la frontera implicaría, al aumentar la dotación de tierra respecto a los otros factores, un aumento de la producción de bienes intensivos en tierra y un deterioro del precio de éstos en relación a bienes intensivos en otros factores. Lo anterior tendría efectos redistributivos: el resultado de un corrimiento de la frontera implicaría, todo lo demás igual, un incremento en la relación salario/renta, pues el trabajo se ha vuelto un factor escaso respecto a la tierra.

El efecto combinado sobre la retribución a los factores en un país abundante en tierra que expande su frontera a la vez que se integra al mercado mundial es difícil de prever. Por un lado, como economía abierta estría sujeta a la dinámica destacada por el teorema Heckscher-Ohlin. Por otro, ante una expansión relativamente rápida de la frontera, sería influida por el efecto Rybczynski.

Así ocurre, por ejemplo, con Australia. Aunque tradicionalmente se le señala como un caso típico de aplicación del teorema Heckscher-Ohlin y sus consecuencias en la relación salario/renta, la evidencia sensible a las diferencias regionales muestra que la evolución de la desigualdad durante la primera globalización en aquel país-continente fue más compleja que lo tradicionalmente aceptado ${ }^{11}$. Mientras la región de Victoria - tradicionalmente considerada representativa de todo el país-, muestra una tendencia al deterioro de la relación salario/renta coincidente con el teorema Heckscher-Ohlin; South Australia —región abundante en tierra y que destaca por sus exportaciones agrícolas - muestra la tendencia inversa. Allí se observa un aumento de la relación salario/ renta entre 1870 y 1890, lo que contradice la predicción que se deriva del teorema Stolper-Samuelson ${ }^{12}$.

En resumen, la expansión de la economía atlántica, motivada por las oportunidades económicas y posibilitada por las innovaciones tecnológicas a que aquella se asocia, puede tener efectos diferentes para la distribución del ingreso a los predichos por el teorema Stolper-Samuelson. Ello es particularmente apreciable en procesos de expansión de la frontera ${ }^{13}$. En ese caso, la propia globalización, entendida en un sentido más amplio que la convergencia de precios, modifica la dotación de factores y los incentivos para explotarlos. La abundante literatura histórica que aplica el modelo Heckscher-Ohlin asume que los factores están dados, cuando una consecuencia de la globalización es justamente la expansión hacia nuevas regiones e incorporación de nuevos recursos. Si esta incorporación hace más escaso el trabajo en

Rybczynski (1955), §9 - §11.

11 También en Canadá existe un comportamiento regional dispar asociado a la incorporación de tierras en el este Emery, Inwood \& Thille (2007).

12 Shanahan \& Wilson (2007), Fig. 3.

13 Harley (2007), p. 245. 
relación a la tierra entonces su retribución debería mejorar en relación a aquella, conduciendo así a una mejora en la distribución del ingreso.

\section{Crecimiento liderado por las exportaciones}

En el período 1879-1913, entre la Guerra del Pacífico y la Primera Guerra Mundial, el PBI chileno creció a una tasa anual de 3,2\%, su población lo hizo al 1,3\%, y el producto por habitante al $1,9 \%^{14}$. Se trata de un ciclo crecimiento - el segundo luego de la Independencia - liderado por las exportaciones. Éstas crecieron un 5\% anual en términos reales para el mismo período y prácticamente duplican su importancia respecto al conjunto de la economía, pasando del 13\% al 24\% del PBI entre 1880 y 1905. El conjunto del comercio exterior creció del $21 \%$ al $47 \%$ en el mismo período $(\text { Gráfico } 1)^{15}$.

\section{GRÁFICO 1}

CRECIMIENTO ECONÓMICO LIDERADO POR LAS EXPORTACIONES (1879-1913)

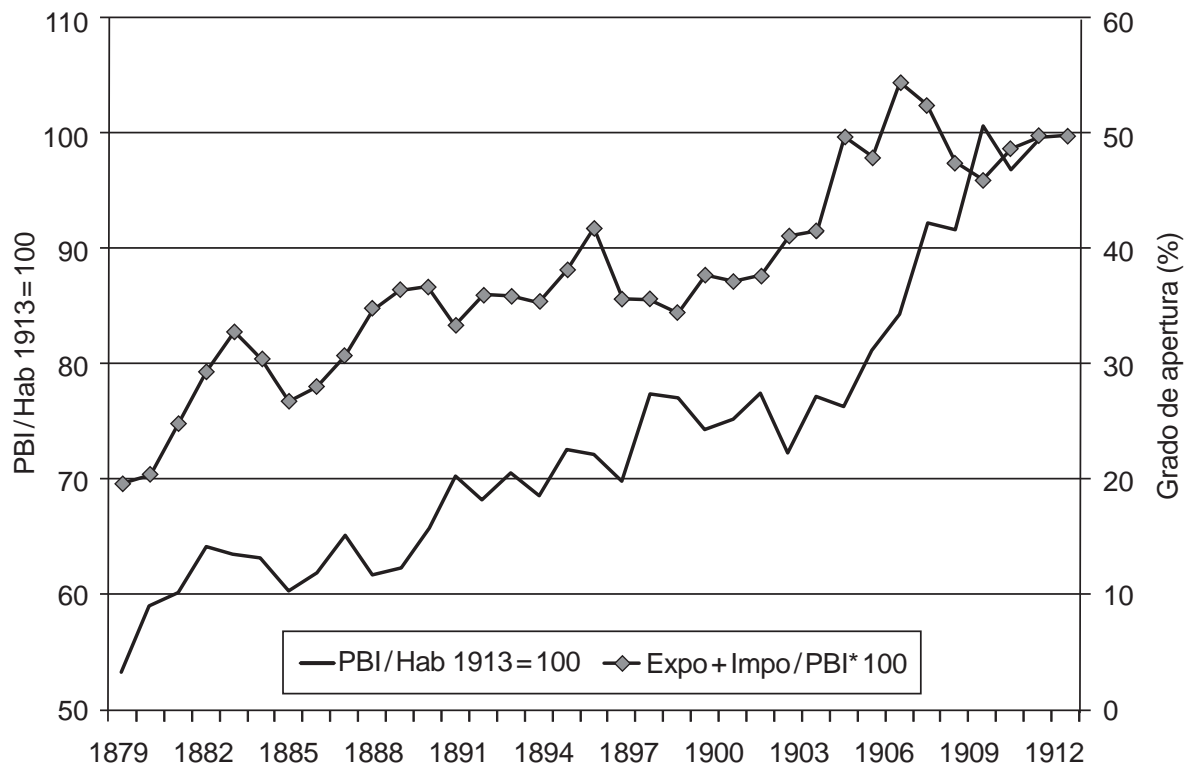

Fuente: Braun et al. (2000), Tablas 1.1 y 5.4. Índice de PBI por habitante $(1913=100)$ y grado de apertura (quantum de las exportaciones más importaciones como porcentaje del PBI).

$14 \quad$ Braun et al. (2000), tabla 1.1 .

15 Braun et al. (2000), tablas 5.4, 5.6 y 4.8 . 
La expansión económica de Chile durante el período es resultado de su inserción internacional exportadora de productos primarios, fundamentalmente nitratos. Se trata de un crecimiento liderado por las exportaciones y beneficiado por la commodity lottery. Si bien parte de la literatura dependentista señalaba al boom del salitre como un caso de "enclave", existe un consenso creciente que destaca las vías por las que el crecimiento exportador impactó al conjunto de la economía: transformaciones demográficas, urbanización, crecimiento del sector servicios — particularmente aquellos brindados por el Estado- y mercado para la producción industrial $^{16}$.

En consonancia con el boom en la producción de nitratos, entre 1880 y 1905, las exportaciones del sector minero acrecientan su importancia respecto a las agropecuarias y manufactureras. En conjunto, el proceso supuso una profundización de la especialización en bienes primarios en las exportaciones (Cuadro 1).

CUADRO 1

ESTRUCTURA DEL VALOR DE LAS EXPORTACIONES (\%). PROMEDIOS QUINQUENALES

\begin{tabular}{cccc}
\hline Quinquenio & Mineras & Agropecuarias & Manufacturas \\
\hline $\mathbf{1 8 8 1 - 1 8 8 5}$ & 83 & 12 & 5 \\
$\mathbf{1 8 8 6 - 1 8 9 0}$ & 86 & 11 & 3 \\
$\mathbf{1 8 9 0 - 1 8 9 5}$ & 85 & 13 & 2 \\
$\mathbf{1 8 9 6 - 1 9 0 0}$ & 86 & 11 & 3 \\
$\mathbf{1 9 0 1 - 1 9 0 5}$ & 90 & 8 & 2 \\
\hline
\end{tabular}

Fuente: Promedios calculados a partir de Braun et al. (2000), Tabla 5.7

Según el teorema Stolper-Samuelson que analizáramos más arriba, y dadas las características del crecimiento descritas, es dable esperar un incremento de la desigualdad en este período. Este es el punto de vista defendido por Prados a partir de la evolución del Índice de Williamson para América Latina y en particular para el caso de Chile ${ }^{17}$. Sin embargo, la evidencia empírica utilizada por Prados para el caso chileno presenta serios problemas.

El índice de Williamson es un ratio entre dos índices, uno de ingreso por habitante y otro de salarios no calificados, en el entendido que éstos expresan el ingreso de los sectores más pobres. Una evolución creciente del ratio implicaría que el ingreso medio crece más rápidamente que el salario de quienes perciben menores ingresos, lo que puede interpretarse como un incremento de la desigualdad. Pero el

\footnotetext{
16 Cariola \& Sunkel (1982); Pinto \& Ortega (1990); Meller (1998); Salazar \& Pinto (2002).

17 Prados (2005), p. 15 y (2007), p. 298.
} 
índice de salarios utilizado por Prados para el caso de Chile no es representativo de los trabajadores no calificados. Este autor toma el Índice de Salarios Reales de Braun et al. y allí se indica que para el período 1860-1927 éste se corresponde con el "Índice General de Remuneraciones" de Wagner ${ }^{18}$.

El Índice General de Remuneraciones presentado por Wagner es, para el período 1860-1913, tomado del índice de remuneraciones de funcionarios públicos construido por Rojas en 1982. Entre 1913 y 1929 Wagner utiliza el índice de Rojas con correcciones menores. Sobre el índice de Rojas dice Wagner:

“Este autor [Rojas] distingue seis subconjuntos — categorías - en base al grado de calificación —establecido a priori-, de las respectivas funciones; ellas van desde el grupo A (Presidente de la República, Embajador y Presidente de la Corte Suprema), hasta la categoría H (Portero, Mayordomo, Cartero, Ordenanza y Mensajero). En realidad la clasificación en cuestión sigue de cerca el ordenamiento de las remuneraciones de acuerdo a sus niveles. El índice por categoría es un índice del promedio simple de las remuneraciones de los diversos cargos que la componen. A su vez, el índice general, es un promedio simple de las diversas categorías. (...) es necesario destacar que la categoría $\mathrm{H}_{\text {, }}$ la de los menos calificados, no incluye: "jornaleros, camineros y soldados rasos", o sea, y con la eventual excepción de los soldados, el trabajo de menor calificación relativa" ${ }^{19}$.

De modo que el índice de salario real utilizado por Prados para construir el índice de Williamson es un promedio no ponderado de seis categorías de trabajadores estatales de las cuales sólo una corresponde a los no calificados -e incluso ésta deja fuera a los de menor calificación. Así, el componente del índice de Williamson construido por Prados que debiera reflejar la evolución del ingreso de los trabajadores de menor calificación refleja otra cosa. Por una parte por tratarse de trabajadores estatales, cuyas remuneraciones se definen de manera distinta que en el caso de los trabajadores de la esfera privada. Pero aún más importante, por ser un promedio no ponderado entre seis categorías, de las cuales solo una corresponde a trabajadores de escasa calificación. El índice de salarios reales utilizado por Prados representa la evolución del ingreso de los funcionarios públicos de calificación media y alta. Por tanto, el índice de Williamson de Chile utilizado por Prados es difícil de interpretar y puede incluso significar lo contrario de lo que sostiene su autor ${ }^{20}$.

Braun et al. (2000), p. 98; Wagner (1992).

Wagner (1992), pp. 54-55, subrayado nuestro.

Prados (2005, 2007). 


\section{Nuestras estimaciones}

El procedimiento general que hemos seguido para estimar la desigualdad personal ha sido construir dos matrices con categorías ocupacionales, una de perceptores de ingresos y otra de ingreso anual corriente. Así, hemos construido cinco bases de datos, las que se clasifican en dos tipos ${ }^{21}$.

Por un lado, una base de datos de panel de categorías de perceptores e ingresos anuales para el período 1860-1930. En ella se mantienen las mismas categorías a lo largo del período, lo que permite brindar información homogénea a costa de cierta rigidez. Toda la información que se presente en forma de series anuales ha sido estimada a partir de esta base. Por otro, cuatro bases distintas para los años 1875, 1885, 1907 y 1930. Ellas son independientes entre sí, de forma que difieren las categorías y son por tanto más sensibles a la estructura de los perceptores de ingreso de cada año. Una ventaja adicional, es que al utilizarse datos censales desagregados a nivel provincial, es posible la estimación de desigualdad por regiones. Usaremos la información que brinden estas bases para controlar los resultados de la serie anual, y para los análisis de desigualdad regional y sectorial.

Las bases representan a la población «con profesión», no a la población total. Suponemos asimismo que existe equivalencia entre la población con profesión y aquellos que reciben ingresos por trabajo o propiedad. El resultado es un análisis de la distribución personal entre perceptores de ingresos y funcional entre propietarios —de tierra y/o capital—y trabajadores. Nuestras bases no permiten realizar análisis por hogares ${ }^{22}$.

\subsection{Perceptores de ingreso}

La principal fuente para construir la base de perceptores de ingreso es la información de población «con profesión» recogida en los censos de población y publicada como apéndice al trabajo de Gálvez y Bravo $^{23}$.

Sin embargo, los datos sin procesar son de poca utilidad debido a los cambios en los criterios de recolección y presentación de la información censal a lo largo del tiempo ${ }^{24}$.

\footnotetext{
$21 \quad$ Esta metodología se adapta de Bertola (2005).

22 Rodríguez Weber (2009). La tesis se encuentra en proceso de publicación. En tanto, el lector interesado puede solicitar una copia al autor que se la enviará con gusto.

23 Gálvez y Bravo (1992).

24 Una crítica de la información censal sobre el tema puede verse en Bauer (1994), Hutchinson (2000), y las propias Gálvez \& Bravo (1992). La forma en que el problema afecta nuestras estimaciones se aborda en Rodríguez Weber (2009).
} 
Si bien la extensión de este artículo nos impide brindar una exposición detallada de los procedimientos seguidos, consideramos ilustrativo hacerlo para alguna de las categorías más importantes de la base de datos utilizada para la estimación anual del período 1860-1930.

\subsubsection{Agricultura}

La categoría peones y gañanes se construyó mediante la agregación de las «profesiones» pescadores, carboneros, gañanes, arrieros y leñadores.

La cantidad de labradores se toma directamente de Salazar, quien presenta una estimación de esta categoría que incluye a los inquilinos de las Haciendas, y minifundistas de diverso tipo ${ }^{25}$.

Los censos de población presentan a la totalidad de los propietarios o tenedores de tierra como «agricultores», lo que incluye a nuestra categoría de labradores. Por tanto, la cantidad de terratenientes se obtiene restando la cifra de labradores a la de agricultores. Los terratenientes se desagregaron según el tamaño de su propiedad estimando siete categorías. Se tomó 1861 como año base ${ }^{26}$. La evolución hasta el año 1907 de las categorías así obtenidas se proyectó según la evolución de la propiedad de la tierra en Chile Central reportada por Bengoa, quien distingue en grandes, medianas y chicas ${ }^{27}$. La cantidad de terratenientes incluidos en las categorías 1 y 2 crece según la evolución de las grandes propiedades, los de las categorías 3 y 4 lo hacen según la evolución de las medianas, y los de las categorías 5, 6 y 7 según la evolución de las chicas. Así, se mantienen las mismas categorías de terratenientes a lo largo del tiempo, pero su crecimiento es sensible a los distintos ritmos que reporta Bengoa para las distintas categorías de propiedades.

\subsubsection{Minería}

Se parte de los datos de Gálvez y Bravo $^{28}$. Para el cálculo de los patrones mineros entre los años 1860 a 1895, se asume que la categoría censal "mineros" se corresponde con los empresarios. Para 1907, cuando bajo la profesión mineros se incluye a todos los integrantes del sector, se utiliza la proporción de patrones que se reporta en el censo de 1930. Los empleados se estiman, para todos los años, a partir de

\footnotetext{
Salazar (1985), cuadro 2.

Tornero (1872), p. 427.

Bengoa (1990), cuadro 1.

Gálvez y Bravo (1992).
} 
la proporción que ocupan en el censo de 1930. Los obreros se calculan como la diferencia entre el conjunto de la fuerza de trabajo y la suma de empresarios y empleados.

\subsubsection{Industria y manufacturas}

Para los Industriales se utiliza la cantidad de establecimientos industriales ${ }^{29}$.

Los oficios recogidos por los censos se agrupan en categorías de perceptores según muestra el Cuadro 2.

\section{CUADRO 2}

CONSTRUCCIÓN DE CATEGORÍAS DE OBREROS Y ARTESANOS

\begin{tabular}{lc}
\hline \multicolumn{1}{c}{ Categorías de Perceptores } & «Profesiones» incluidas en las categorías \\
\hline Artesanos textiles baja calificación & $\begin{array}{c}\text { Hilanderas y tejedoras, tejedoras, curtidores, } \\
\text { pelloneros y montureros, costureras, riendereos } \\
\text { y talabarteros, }\end{array}$ \\
Artesanos textiles calificados & $\begin{array}{c}\text { Bordadores, miñaqueras, modistas, sastres } \\
\text { sombrereros, tapiceros }\end{array}$ \\
Oficios madera y afines baja calificación & Aserradores, carpinteros, escoberos y canasteros \\
Oficios madera y afines, alta calificación & Toneleros, ebanistas y talladores, carroceros \\
Oficios metalúrgicos, baja calificación & Herradores, herreros, hojalateros, plomeros \\
Oficios metalúrgicos y construcción, alta & Torneros, fundidores, mecánicos, plateros, vidrieros \\
calificación & y electricistas \\
Construcción y artículos del hogar, baja & Alfareros y loceros, jaboneros, veleros, vaseros y \\
calificación & vasijeros, albañiles, pintores \\
Alimentos y afines & Cigarreros, confiteros y pasteleros, dulceros \\
& lecheros, panaderos, carniceros, queseros \\
Otros baja calificados & Zapateros y peluqueros \\
\hline
\end{tabular}

El Cuadro 3 presenta una síntesis del procedimiento seguido en la construcción de las categorías por sector de actividad económica y tipo de ingreso, junto con la proporción que ocupan en el total al inicio $-1860-$ y final -1930 - de la serie.

Díaz et al. (1998), cuadro AE 7. 


\section{CUADRO 3}

CATEGORÍAS OCUPACIONALES POR SECTOR DE ACTIVIDAD ECONÓMICA Y TIPO DE INGRESO. PROPORCIÓN EN EL TOTAL PARA 1860 Y 1930. FUENTES Y PROCEDIMIENTO DE CONSTRUCCIÓN. BASE ANUAL PERÍODO 1860-1930

\begin{tabular}{|c|c|c|c|c|}
\hline Sector & $\begin{array}{l}\% \text { del total } \\
\text { en } 1860\end{array}$ & $\begin{array}{l}\% \text { del total } \\
\text { en } 1930\end{array}$ & Fuentes & Procedimiento \\
\hline Agro & 45,7 & 40,2 & & \\
\hline Terratenientes & 1,9 & 2,5 & $\begin{array}{l}\text { Tornero (1872), Salazar } \\
\text { (1985), Bengoa (1990), } \\
\text { Gálvez y Bravo (1992), } \\
\text { Censo Población (1930), } \\
\text { Censo agrícola (1936) }\end{array}$ & $\begin{array}{l}\text { Desagregación profesión "agri- } \\
\text { cultores", según Tornero (1872) y } \\
\text { Salazar (1985). El crecimiento se } \\
\text { proyecta según Bengoa (1990) y el } \\
\text { Censo agrícola de } 1936 .\end{array}$ \\
\hline Peones y gañanes & 26,3 & 19,7 & $\begin{array}{l}\text { Gálvez y Bravo (1992), } \\
\text { Censo Población (1930) }\end{array}$ & $\begin{array}{l}\text { Agregación de diversas categorías } \\
\text { de peones y trabajadores. }\end{array}$ \\
\hline Labradores & 17,5 & 18 & Salazar (1985) & $\begin{array}{l}\text { Corresponde a la cantidad de } \\
\text { Labradores de Salazar (1985, } \\
\text { Cuadro } 2 ; 36)\end{array}$ \\
\hline Minería & 3,3 & 6,2 & & \\
\hline Mineros & 0,01 & 0,01 & $\begin{array}{l}\text { Gálvez y Bravo (1992), } \\
\text { Censo Población (1930) }\end{array}$ & $\begin{array}{l}\text { Se toma la información de la cate- } \\
\text { goría Mineros para algunos años } \\
\text { en que está desagregada. Para } \\
\text { los años que se presenta todo el } \\
\text { sector bajo esta categoría, ( } 1907 \\
\text { y 1920) se desagrega según la } \\
\text { proporción de patrones del Censo } \\
\text { de } 1930 .\end{array}$ \\
\hline Empleados & 0,3 & 0,6 & $\begin{array}{l}\text { Gálvez y Bravo (1992), } \\
\text { Censo Población (1930) }\end{array}$ & $\begin{array}{l}\text { Se mantiene proporción de } 1930 \\
\text { para toda la serie. }\end{array}$ \\
\hline Obreros & 3 & 5,6 & $\begin{array}{l}\text { Gálvez y Bravo (1992), } \\
\text { Censo Población (1930) }\end{array}$ & $\begin{array}{l}\text { Agregación de diversas categorías } \\
\text { de trabajadores de minería. }\end{array}$ \\
\hline Industria & 31,8 & 24,4 & & \\
\hline Industriales & 0,4 & 1 & Díaz, et al. (1998) & $\begin{array}{l}\text { Número de "establecimientos } \\
\text { industriales". }\end{array}$ \\
\hline Artesanos textiles & 22,9 & 7,9 & Gálvez y Bravo (1992) & Agregación de diversas categorías. \\
\hline Oficios madera y afines & 2,4 & 3,4 & Gálvez y Bravo (1992) & Agregación de diversas categorías. \\
\hline Metalurgia & 0,6 & 1,4 & Gálvez y Bravo (1992) & Agregación de diversas categorías. \\
\hline $\begin{array}{l}\text { Construcción y } \\
\text { artículos del hogar }\end{array}$ & 1,2 & 1,9 & Gálvez y Bravo (1992) & Agregación de diversas categorías. \\
\hline Alimentos y afines & 1 & 1,6 & Gálvez y Bravo (1992) & Agregación de diversas categorías. \\
\hline $\begin{array}{l}\text { Artesanos textiles } \\
\text { calificados }\end{array}$ & 1 & 1,9 & Gálvez y Bravo (1992) & Agregación de diversas categorías. \\
\hline $\begin{array}{l}\text { Madera y afines } \\
\text { calificados }\end{array}$ & 0,1 & 0,7 & Gálvez y Bravo (1992) & Agregación de diversas categorías. \\
\hline $\begin{array}{l}\text { Metalurgia y } \\
\text { construcción } \\
\text { calificados }\end{array}$ & 0,2 & 2,6 & Gálvez y Bravo (1992) & Agregación de diversas categorías. \\
\hline Otros (no calificados) & 2 & 2 & & Agregación de diversas categorías. \\
\hline
\end{tabular}




\begin{tabular}{|c|c|c|c|c|}
\hline Sector & $\begin{array}{c}\% \text { del total } \\
\text { en } 1860\end{array}$ & $\begin{array}{c}\% \text { del total } \\
\text { en } 1930\end{array}$ & Fuentes & Procedimiento \\
\hline Transporte & 0,6 & 4,2 & & \\
\hline Obreros del ferrocarril & 0,03 & 1,5 & \multirow{4}{*}{$\begin{array}{l}\text { Gálvez y Bravo (1992), } \\
\text { Anuario 1871, Braun et al } \\
\text { (2000), Díaz et al. (2007) }\end{array}$} & \multirow{4}{*}{$\begin{array}{l}\text { Agregación de ferrocarrileros, y } \\
\text { elaboración de categorías pro- } \\
\text { fesionales, empleados y obreros } \\
\text { según proporción de Anuario } 1871 \text {. } \\
\text { Construcción de Choferes y otros } \\
\text { mediante agregación de diversas } \\
\text { categorías. }\end{array}$} \\
\hline $\begin{array}{l}\text { Empleados del } \\
\text { ferrocarril }\end{array}$ & 0,001 & 0,2 & & \\
\hline $\begin{array}{l}\text { Profesionales del } \\
\text { ferrocarril }\end{array}$ & 0,001 & 0,1 & & \\
\hline Conductores y otros & 0,6 & 2,4 & & \\
\hline Estado & 1,9 & 6,1 & & \\
\hline $\begin{array}{l}\text { Funcionarios (ocho } \\
\text { categorías) }\end{array}$ & 0,3 & 1,6 & \multirow{3}{*}{$\begin{array}{l}\text { Gálvez y Bravo (1992), } \\
\text { Censo Población (1930) } \\
\text { Anuario } 1871\end{array}$} & \multirow{3}{*}{$\begin{array}{l}\text { Con las cifras censales se constru- } \\
\text { yen las categorías de funcionarios, } \\
\text { docentes y militares y policías. } \\
\text { Cada una de estas categorías se } \\
\text { desagregan según la estructura } \\
\text { salarial en } 1871 \text { proporcionada por } \\
\text { el anuario. }\end{array}$} \\
\hline $\begin{array}{l}\text { Docentes (seis } \\
\text { categorías) }\end{array}$ & 0,2 & 1,6 & & \\
\hline $\begin{array}{l}\text { Militares y policías } \\
\text { (siete categorías) }\end{array}$ & 1,4 & 2,9 & & \\
\hline Servicios Personales & 16,7 & 18,9 & & \\
\hline Profesionales & 0,2 & 1,2 & $\begin{array}{l}\text { Gálvez y Bravo (1992), } \\
\text { Braun et al (2000), Díaz } \\
\text { et al. (2007) }\end{array}$ & $\begin{array}{l}\text { Agregación de diversas catego- } \\
\text { rías censales. A partir de } 1920 \text { se } \\
\text { proyecta por serie de trabajo de } \\
\text { "resto" según Braun et al (2000). }\end{array}$ \\
\hline $\begin{array}{l}\text { Sirvientes y peones } \\
\text { urbanos }\end{array}$ & 16,5 & 17,7 & $\begin{array}{l}\text { Gálvez y Bravo (1992), } \\
\text { Braun et al (2000), Díaz } \\
\text { et al. (2007) }\end{array}$ & $\begin{array}{l}\text { Agregación de diversas catego- } \\
\text { rías censales. A partir de } 1920 \text { se } \\
\text { proyecta por serie de trabajo de } \\
\text { "resto" según Braun et al (2000). }\end{array}$ \\
\hline
\end{tabular}

\subsection{Ingresos anuales corrientes}

Al igual que en el caso anterior, pasamos a detallar el procedimiento seguido en la estimación de los ingresos anuales para las categorías más importantes de perceptores.

\subsubsection{Agricultura}

Para las 7 categorías de propietarios se estableció un año base en $1861^{30}$. Luego el ingreso se proyecta por el índice de Precios Agrícolas (IPALS) ${ }^{31}$.

Para los peones y gañanes, se utilizan los datos de salario diario ${ }^{32}$. La fuente los presenta en períodos quinquenales. Aquí asignamos el valor en pesos para el año central del tramo y los años intermedios se completan por interpolación.

Tornero (1871), pp. 425-427.

Wagner (1992).

32 Bauer (1994), cuadro 31. 
Para los labradores, se establece la cifra de 90 pesos anuales hacia $1861^{33}$, y se proyecta según un índice construido al efecto. Éste constituye el promedio de dos índices, el de precios agrícolas IPALS ${ }^{34}$, y la remuneración de los gañanes ${ }^{35}$. Esta decisión se justifica por la situación ambigua de los labradores, cuyo ingreso total derivaba de un salario (con todas las prevenciones que esto significa en el medio rural decimonónico chileno) y del usufructo de un trozo de tierra.

\subsubsection{Minería}

Para la categoría de obreros mineros se tienen ingresos para distintos años. Los períodos intermedios se llenan mediante interpolación.

Para la categoría empleados, dada la ausencia de datos se toma el ingreso de un capataz de industria para el año $1869^{36}$. Dada la información cualitativa disponible, parece una aproximación razonable un ingreso que, como el del capataz, ubica a los empleados de las minas en el $80 \%$ del ingreso de los ingenieros ${ }^{37}$, y 7 veces y media superior al ingreso medio obrero. El conjunto de la serie se proyecta por el Índice General de Remuneraciones ${ }^{38}$.

Para estimar las utilidades de la minería se realiza una estimación del Valor Bruto de Producción (VBP) minero en precios corrientes a partir de series de producción física de cobre, yodo y salitre; series de precios en dólares, y serie de tipo de cambio ${ }^{39}$. Luego se estima el Valor Agregado Bruto (VAB) mediante un coeficiente de $0,75^{40}$. Al VAB se le restan los impuestos captados por el Estado ${ }^{41}$. Al VAB post impuestos se le resta la masa salarial. Se obtiene así la serie Utilidades del capital. Para estimar las utilidades retenidas en Chile, se multiplican las utilidades totales por un coeficiente de la porción de capitales chilenos en la producción salitrera ${ }^{42}$. Finalmente, la masa de utilidades de los capitalistas chilenos se dividen entre la cantidad de patrones mineros a fin de obtener una serie de ingreso personal.

Salazar (1985).

Wagner (1992).

Bauer (1994), cuadro 31.

García (1989).

Rojas (1982).

De Wagner (1992).

Braun et al. (2000); Díaz et al. (1998); Cariola \& Sunkel (1982).

Braun et al. (2000).

Díaz et al. (1998).

Cariola \& Sunkel (1982), cuadro 16. 


\subsubsection{Industria y manufacturas}

Para la estimación del ingreso anual de los trabajadores de la industria y la manufactura se sigue el método ilustrado en el Cuadro 4.

\section{CUADRO 4}

FUENTES Y PROCEDIMIENTO PARA LA ESTIMACIÓN DE INGRESOS DE TRABAJADORES DEL SECTOR INDUSTRIA Y MANUFACTURAS

\begin{tabular}{|c|c|c|c|}
\hline Categoría & $\begin{array}{c}\text { Año y género para } \\
\text { los que hay información }\end{array}$ & Fuentes & $\begin{array}{l}\text { Método para } \\
\text { años faltantes }\end{array}$ \\
\hline (1) & (2) & (3) & (4) \\
\hline $\begin{array}{l}\text { Artesanos textiles baja } \\
\text { calificación }\end{array}$ & $\begin{array}{l}1859(\mathrm{~h}) ; 1870(\mathrm{~m}) ; 1895 \\
(\mathrm{~m}) ; 1905(\mathrm{~h}, \mathrm{~m}) ; 1906(\mathrm{~h}, \mathrm{~m}) ; \\
1910(\mathrm{~m}) ; 1913(\mathrm{~h}) ; 1921(\mathrm{~m}) ; \\
1923(\mathrm{~h}) ; 1930(\mathrm{~h})\end{array}$ & $\begin{array}{l}\text { Álvarez (1936) Anuario } \\
\text { (1871) Censo Industrial } \\
\text { (1895) DeShazo (1983), } \\
\text { Ortiz Letelier (2005) }\end{array}$ & $\begin{array}{l}\text { Los años faltantes se llenan } \\
\text { por Interpolación. }\end{array}$ \\
\hline $\begin{array}{l}\text { Artesanos textiles } \\
\text { calificados }\end{array}$ & $1903(h)$ & Errazúriz y Eizaguirre (1903) & $\begin{array}{l}\text { La serie se proyecta según } \\
\text { evolución de metalúrgicos } \\
\text { calificados }\end{array}$ \\
\hline $\begin{array}{l}\text { Oficios madera y afines } \\
\text { baja calificación }\end{array}$ & $\begin{array}{l}1859(h) ; 1869(h) ; 1893(h) \\
1895(h), 1913(h) 1923(h) \\
1930(h)\end{array}$ & $\begin{array}{l}\text { Errazúriz y Eizaguirre (1903) } \\
\text { Álvarez (1936), García } \\
\text { (1989) }\end{array}$ & $\begin{array}{l}\text { Interpolación para años } \\
\text { faltantes Mujeres como } 50 \% \\
\text { de hombres. }\end{array}$ \\
\hline $\begin{array}{l}\text { Oficios madera y afines, } \\
\text { alta calificación }\end{array}$ & $1869(h) 1893(h)$ & $\begin{array}{l}\text { Errázuriz y Eizaguirre (1903) } \\
\text { García (1989) }\end{array}$ & $\begin{array}{l}\text { Antes de } 1869 \text {, y luego de } \\
1893 \text { se proyecta según } \\
\text { Metalurgia calificados. Entre } \\
1869 \text { y } 1893 \text { por interpola- } \\
\text { ción. Mujeres como } 50 \% \text { de } \\
\text { hombres. }\end{array}$ \\
\hline $\begin{array}{l}\text { Oficios metalúrgicos, baja } \\
\text { calificación }\end{array}$ & $\begin{array}{l}\text { 1859(h); 1869(h); 1893(h); } \\
1895(\mathrm{~h}) ; 1902(\mathrm{~h}) ; 1906(\mathrm{~h}) \\
1913(\mathrm{~h}) ; 1923(\mathrm{~h}) ; 1930(\mathrm{~h})\end{array}$ & $\begin{array}{l}\text { Errazúriz y Eizaguirre } \\
\text { (1903) Álvarez (1936), Ortiz } \\
\text { Letelier, García (1989) }\end{array}$ & $\begin{array}{l}\text { Datos faltantes se llenan por } \\
\text { interpolación. Mujeres como } \\
\text { un } 50 \% \text { de hombres. }\end{array}$ \\
\hline $\begin{array}{l}\text { Oficios metalúrgicos } \\
\text { y construcción, alta } \\
\text { calificación }\end{array}$ & $\begin{array}{l}1860(h) ; 1893(h) ; 1895(h) ; \\
1913(h) ; 1923(h) ; 1930(h)\end{array}$ & $\begin{array}{l}\text { Errazúriz y Eizaguirre (1903) } \\
\text { Álvarez (1936), García } \\
\text { (1989) }\end{array}$ & $\begin{array}{l}\text { Datos faltantes se llenan por } \\
\text { interpolación. Mujeres como } \\
\text { un } 50 \% \text { de hombres. }\end{array}$ \\
\hline $\begin{array}{l}\text { Construcción y artículos } \\
\text { del hogar, baja calificación }\end{array}$ & $\begin{array}{l}1859(h) 1893(h) ; 1895(h) ; \\
1906(h) ; 1913(h) ; 1923(h) ; \\
1930(h)\end{array}$ & $\begin{array}{l}\text { Errazúriz y Eizaguirre } \\
\text { (1903) Álvarez (1936), Ortiz } \\
\text { Letelier; García }\end{array}$ & $\begin{array}{l}\text { Datos faltantes se llenan por } \\
\text { interpolación. Mujeres como } \\
\text { un } 50 \% \text { de hombres. }\end{array}$ \\
\hline Alimentos y afines & $1869(h) ; 1893(h) ; 1906(h)$ & $\begin{array}{l}\text { Errazúriz y Eizaguirre } \\
\text { (1903), Ortiz Letelier, García } \\
\text { (1989) }\end{array}$ & $\begin{array}{l}\text { Datos faltantes se llenan por } \\
\text { interpolación. Mujeres como } \\
\text { un } 50 \% \text { de hombres. Antes } \\
\text { de } 1869 \text { y luego de } 1906 \text { se } \\
\text { mueve según textiles }\end{array}$ \\
\hline Otros baja calificados & $\begin{array}{l}\text { 1859(h); 1893(h); 1913(h); } \\
1923(h) ; 1930(h)\end{array}$ & Álvarez (1936) & $\begin{array}{l}\text { Datos faltantes se llenan por } \\
\text { interpolación. Mujeres como } \\
\text { un } 50 \% \text { de hombres. }\end{array}$ \\
\hline
\end{tabular}


Para los Industriales se toma la masa de utilidades para los años 1915-1918 ${ }^{43}$. Se la divide por el total estimado de industriales para obtener el ingreso per cápita. Para los años 1860-1914 y 1919-1930, se toman los valores de 1915 y 1918 y a partir de allí se proyecta por un índice de Ingreso de Industriales. Este se construye como el promedio de dos índices. El primero refleja la evolución del producto por establecimiento industrial $^{44}$. El segundo, la relación existente entre el Valor Agregado Bruto industrial ${ }^{45}$ y la masa salarial de nuestra estimación. De esta forma el índice de Ingreso de Industriales es sensible tanto a la evolución de la producción por establecimiento industrial, como a los eventuales cambios en la proporción de la masa salarial dentro en el total de la producción. El Cuadro 5 presenta una descripción básica del procedimiento seguido y las fuentes utilizadas en todas las categorías.

CUADRO 5

FUENTE Y PROCEDIMIENTO UTILIZADO EN LA ESTIMACIÓN DEL INGRESO ANUAL PARA EN CADA CATEGORÍA DE PERCEPTORES

\begin{tabular}{|c|c|c|}
\hline $\begin{array}{c}\text { Categoría } \\
\text { ocupacional }\end{array}$ & Fuentes & Procedimiento \\
\hline Terratenientes & $\begin{array}{l}\text { Tornero (1872), } \\
\text { Wagner (1992) }\end{array}$ & $\begin{array}{l}\text { A partir del ingreso del año base (1861), tomado de Tornero } \\
\text { (1872), la serie de ingresos se proyecta por Índice de precios } \\
\text { agrícola IPALS, tomado de Wagner (1992). }\end{array}$ \\
\hline Labradores & $\begin{array}{l}\text { Salazar (1985), } \\
\text { Bauer (1994), } \\
\text { Díaz et al. (2007), } \\
\text { Wagner (1992), } \\
\text { Bengoa (1990) }\end{array}$ & $\begin{array}{l}\text { Se parte de un año base (1861) tomado de Salazar (1985), y se } \\
\text { proyecta mediante un Índice construido ad hoc como el prome- } \\
\text { dio del Índice de precios agrícolas, y la evolución del salario de } \\
\text { gañanes y peones. }\end{array}$ \\
\hline Peones y gañanes & $\begin{array}{l}\text { Bauer (1994), } \\
\text { Díaz et al. (2007), } \\
\text { Wagner (1992), } \\
\text { Bengoa (1990) }\end{array}$ & $\begin{array}{l}\text { Estimación de salario diario presentada en tramos de cinco } \\
\text { años de Bauer (1994) por días trabajados según Wagner (1992) } \\
\text { y Díaz et al. }(2007)^{46} \text {. Para los períodos inter-quinquenales se } \\
\text { realiza interpolación. }\end{array}$ \\
\hline Mineros (patrones) & $\begin{array}{l}\text { Braun et al (2000), } \\
\text { Díaz et al. (1998 y 2007), } \\
\text { Cariola \& Sunkel (1983), } \\
\text { Wagner et al (2000) }\end{array}$ & $\begin{array}{l}\text { Se construye una estimación de valor agregado minero a } \\
\text { precios corrientes (cobre, yodo y salitre). Se restan impuestos } \\
\text { y masa salarial para obtener una estimación de utilidades. Se } \\
\text { multiplican las utilidades por un coeficiente que varía según la } \\
\text { participación de capitales chilenos en la explotación de salitre. } \\
\text { Se obtiene una serie de utilidades en manos de capitalistas } \\
\text { chilenos. Las utilidades de capitalistas chilenos se dividen entre } \\
\text { la estimación de Patrones mineros. }\end{array}$ \\
\hline
\end{tabular}

43 Carmagnani (1998), cuadro 11.

$44 \quad$ Díaz et al. (1998).

45 Díaz et al. (1998).

46 La permanencia de una misma cantidad de días trabajados a lo largo del período es discutible. En opinión de Bauer (1994), los peones trabajaban menos días al año en el siglo xix que en el xx. Salazar (1985), por su parte, estima una cantidad de días trabajados por peón muy inferior a la manejada por Wagner (1992) para el conjunto de la fuerza de trabajo. Aquí se ha preferido usar la estimación de Wagner a fin de hacer la serie homogénea, y no introducir por esa vía un sesgo en la evolución de la desigualdad, aunque el procedimiento es obviamente discutible. 


\begin{tabular}{|c|c|c|}
\hline $\begin{array}{c}\text { Categoría } \\
\text { ocupacional }\end{array}$ & Fuentes & Procedimiento \\
\hline Mineros (empleados) & $\begin{array}{l}\text { García (1989), } \\
\text { Wagner (1992) }\end{array}$ & $\begin{array}{l}\text { Se proyecta el salario de un capataz de industria para el año } \\
1869 \text { por el Índice General de Remuneraciones (IGR) corregido } \\
\text { por IPC de Wagner (1992). }\end{array}$ \\
\hline Mineros (obreros) & $\begin{array}{l}\text { Salazar (1985), } \\
\text { Ortiz Letelier (2005) }\end{array}$ & $\begin{array}{l}\text { Se toma información para diversos años (1860-1879; } 1904 \text {, } \\
1916 \text { y 1919) Los periodos intermedios se completan mediante } \\
\text { interpolación. }\end{array}$ \\
\hline Industriales & $\begin{array}{l}\text { Carmagnani (1998), } \\
\text { Braun et al (2000), } \\
\text { Díaz et al. (1998 y 2007) }\end{array}$ & $\begin{array}{l}\text { Se establecen dos años base (1915 y 1918) a partir de informa- } \\
\text { ción sobre utilidades industriales proporcionadas por Carmag- } \\
\text { nani (1998). Los períodos } 1860-1914 \text { y } 1919-1930 \text { se proyectan } \\
\text { por un Índice de ingresos industriales construido al efecto que } \\
\text { tiene en cuenta la evolución del producto por establecimiento y } \\
\text { la relación entre el VAB industrial y la masa salarial. }\end{array}$ \\
\hline $\begin{array}{l}\text { Obreros y artesanos } \\
\text { industriales }\end{array}$ & $\begin{array}{l}\text { Álvarez (1936), } \\
\text { Anuario (1871), } \\
\text { Censo Industrial (1895), } \\
\text { Ortiz Letelier (2005), } \\
\text { Errazúriz y Eizaguirre (1903), } \\
\text { Díaz et al (2007) }\end{array}$ & $\begin{array}{l}\text { Se parte de información para diversos años de diversas catego- } \\
\text { rías. Los años faltantes se completan por interpolación. }\end{array}$ \\
\hline Transporte & $\begin{array}{l}\text { Anuario (1871), } \\
\text { Errazúriz y Eizaguirre (1903), } \\
\text { García (1989), } \\
\text { Wagner (1992), } \\
\text { Díaz et al (2007) }\end{array}$ & $\begin{array}{l}\text { Se construyen estimaciones de ingreso para años base de } \\
\text { las tres categorías de ferrocarrileros a partir de la información } \\
\text { presente en el Anuario (1871). Esta se proyecta para el período } \\
1891-1924 \text { mediante Índice de ingresos de profesionales, em- } \\
\text { pleados y obreros de ferrocarril de Wagner (1992), Ilenando los } \\
\text { años faltantes por interpolación. Para el período 1860-1890, y } \\
\text { 1925-1930, se proyecta por el Índice General de Remuneracio- } \\
\text { nes (Wagner 1992) corregido por IPC. }\end{array}$ \\
\hline $\begin{array}{l}\text { Estado: funcionarios, } \\
\text { docentes, militares y } \\
\text { policías }\end{array}$ & $\begin{array}{l}\text { Anuario (1871), } \\
\text { Díaz et al (2007) }\end{array}$ & $\begin{array}{l}\text { Se toma el ingreso promedio de cada categoría en el año } 1870 \\
\text { según información del anuario. Luego se proyectan según los } \\
\text { Índices de ingresos de funcionarios estatales de Rojas, citados } \\
\text { en Díaz et al (2007). }\end{array}$ \\
\hline Profesionales & Díaz et al. (2007) & $\begin{array}{l}\text { Se utiliza la serie anual del ingreso de Ingenieros de Rojas, } \\
\text { citada en Díaz et al (2007). }\end{array}$ \\
\hline Sirvientes & $\begin{array}{l}\text { Salazar (1985), } \\
\text { Anuario (1871), } \\
\text { Errazúriz y Eizaguirre (1903), } \\
\text { Álvarez (1936), } \\
\text { Díaz et al (2007) }\end{array}$ & $\begin{array}{l}\text { Se tiene información para diversos años. El resto se completa } \\
\text { mediante interpolación. }\end{array}$ \\
\hline
\end{tabular}

\subsection{Medidas de desigualdad}

A partir de las bases de datos de cantidad de perceptores y de ingresos anuales corrientes de cada categoría ocupacional, se estimó una serie de Ingreso Interior Bruto (YIB) en precios corrientes. La misma, una vez transformada a valores constantes mediante el uso de distintos deflactores, muestra un crecimiento anual similar a la serie de Valor Agregado Bruto ${ }^{47}$ y presenta con ésta un coeficiente de correlación de

$47 \quad$ Díaz et al. (1998) 
0,98 ${ }^{48}$. También se estimó una serie de Ingreso Nacional Bruto (YNB) como la diferencia entre el YIB y una estimación de la porción del Excedente de Explotación captado por las inversiones extranjeras en la producción del salitre, cuyo procedimiento de estimación fue descrito al explicar la estimación de los ingresos de los patrones mineros. Los índices de distribución personal utilizados —Gini, GE (0) y GE (1) - se calcularon a partir de la estimación del YNB, en tanto la evolución de los ratios entre utilidades y salarios se estimaron a partir del YIB.

\section{5. ¿Qué dice la nueva evidencia?}

Nuestras estimaciones de desigualdad no son congruentes con el resultado predicho por el teorema Stolper-Samuelson. Nuestros índices muestran una tendencia al descenso entre 1875 y 1885 (Cuadro 6), y una mejora importante de la distribución del ingreso entre 1895 y 1905 (Gráfico 2).

Es decir que a medida que se profundiza la especialización en bienes intensivos en recursos naturales la distribución del ingreso mejora en lugar de empeorar. Esta mejora se produce en dos fases. La primera - que se aprecia mejor en los índices de entropía presentados en el Cuadro 6 y el Gráfico 2-, transcurre desde 1875/80 hasta 1888/90. La segunda, desde 1894 hasta 1903, presenta una caída más importante (Gráfico 2). En el ínterin se produce un aumento de la desigualad de distinta intensidad según el índice.

En lo que resta del artículo nos proponemos mostrar que esta mejora en la distribución del ingreso es consecuencia en parte del impacto redistributivo que tuvo la incorporación de nuevos territorios. La expansión de la frontera modificó la dotación de factores abriendo un proceso de cambio estructural e institucional que amplió las posibilidades de la fuerza de trabajo no calificada. Esta se movilizó hacia las nuevas regiones y vio incrementarse su ingreso, lo que provocó una caída en la desigualdad.

CUADRO 6

ÍNDICES DE DESIGUALDAD PARA AÑOS SELECCIONADOS

\begin{tabular}{cccc}
\hline Año & GE (0) & GE (1) & GINI \\
\hline $\mathbf{1 8 7 5}$ & 0,49 & 1,01 & 0,52 \\
$\mathbf{1 8 8 5}$ & 0,42 & 0,70 & 0,49
\end{tabular}

Fuente: Estimación propia. Calculado a partir de estimación del Ingreso Nacional Bruto.

48 Bertola \& Rodríguez Weber (2009). 


\section{GRÁFICO 2}

ÍNDICES DE DESIGUALDAD PERSONAL (1880-1905)

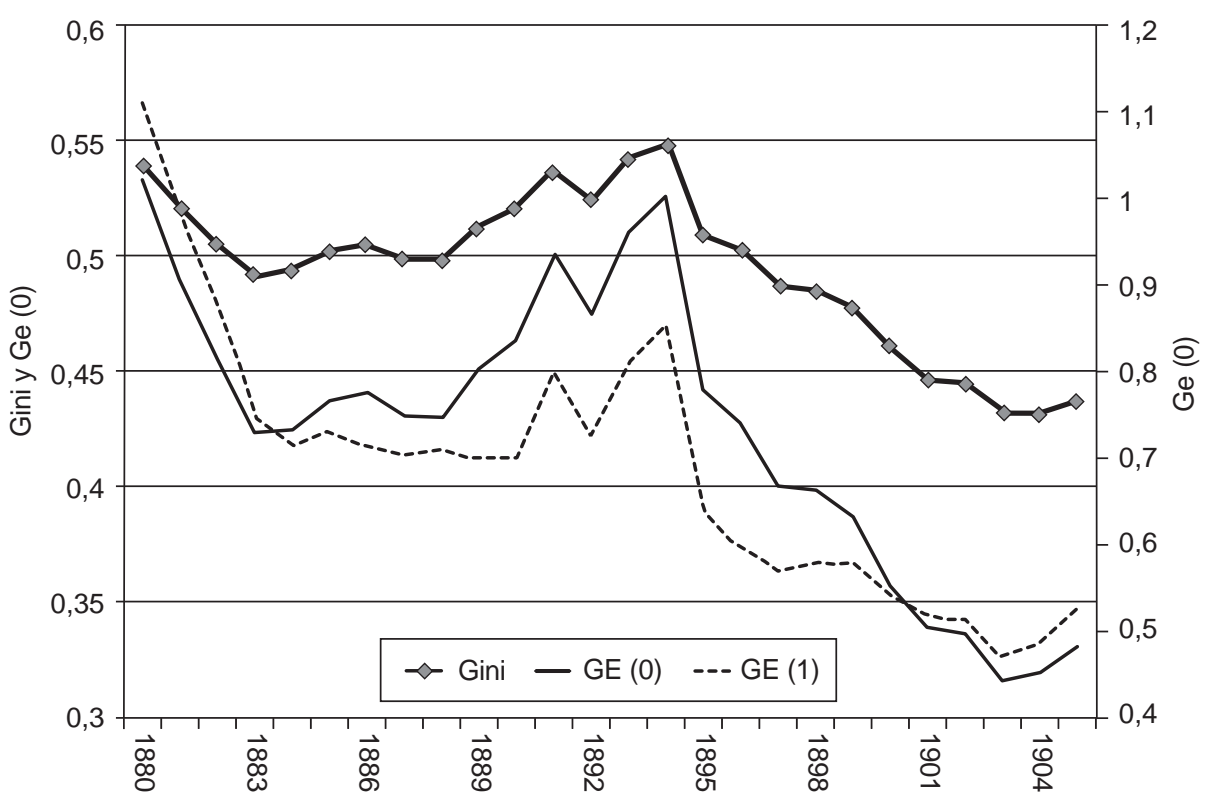

Fuente: Estimación propia. Calculado a partir de estimación del Ingreso Nacional Bruto.

\section{Expansión de la frontera, migraciones y desigualdad.}

De acuerdo con Rybczynski el cambio relativo en la dotación de factores conduce al deterioro del precio relativo del bien intensivo en el factor que aumenta ${ }^{49}$. Ello debería conducir a un descenso de la retribución al factor que se ha hecho relativamente más abundante, en relación a la retribución al factor ahora relativamente más escaso. En consecuencia, una expansión de la frontera que acrecienta la dotación de tierra y la vuelve más abundante en relación al trabajo, conduciría a un aumento de la relación salario/renta, y supondría una mejora en los indicadores de desigualdad. Esto fue lo que ocurrió en Chile entre 1880 y 1905. 


\subsection{Expansión de la frontera y dotación de factores}

Ni las regiones salitreras al norte, ni la Araucanía al sur constituían territorios vacíos y ambas regiones tenían vínculos con Chile antes de 1880. Sin embargo, lo cierto es que es durante ésa década que ambos se incorporan efectivamente al sistema económico chileno modificando sus proporciones factoriales.

La expansión hacia el Norte que diera lugar al boom salitrero fue posible debido a la victoria militar chilena en la Guerra del Pacífico -también llamada del salitreante Perú y Bolivia. A ésta siguió otra al sur sobre la población aborigen, que permitió la "pacificación" de la Araucanía. Ambas victorias significaron la integración efectiva al territorio chileno de algo más de un tercio de su superficie de entonces ${ }^{50}$. La incorporación supuso además un enriquecimiento sustancial en recursos naturales, en salitre al norte y tierras agrícolas y forestales al sur. Según Cariola \& Sunkel, la expansión de la frontera debida a la incorporación de estas regiones constituye, junto con las transformaciones del Valle Central a ella asociadas, el rasgo central del ciclo salitrero ${ }^{51}$.

El Cuadro 7 presenta una estimación del cambio en la dotación relativa de los factores, usando la evolución de la cantidad de superficie territorial $\left(\mathrm{km}^{2}\right)$ por habitante como aproximación al mismo.

CUADRO 7

UNA ESTIMACIÓN DEL IMPACTO DE LA EXPANSIÓN DE LA FRONTERA EN LA DOTACIÓN RELATIVA DE TRABAJO Y TIERRA

\begin{tabular}{lcccc}
\hline & $\mathbf{1 8 7 5}$ & $\mathbf{1 8 8 5}$ & $\mathbf{1 8 9 5}$ & $\mathbf{1 9 0 7}$ \\
\hline Superficie $\left(\mathbf{k m}^{\mathbf{2}}\right)$ & 371.385 & 585.824 & 585.824 & 585.824 \\
Población & 2.074 .055 & 2.527 .304 & 2.802 .145 & 3.349 .279 \\
$\mathbf{K m}$ por habitante & 0,18 & 0,23 & 0,21 & 0,18 \\
$\mathbf{K m}$ por habitante & 100 & 129 & 117 & 98 \\
$\quad$ Índice $\mathbf{1 8 7 5}=\mathbf{1 0 0}$ & & & & \\
\hline
\end{tabular}

Fuente: La información se extrae del Censo de Población de 1907. Para el año 1875 se tiene en cuenta el total de la población, dividido el total de la superficie, menos la correspondiente a las que en 1907 serían las provincias de Tacna, Tarapacá y Antofagasta al norte; y Malleco y Cautín al sur, las que se incorporan en la estimación a partir de 1885. No se tiene en cuenta el territorio de Magallanes que se mantiene virtualmente despoblado para todo el período siendo entre 0,1 y $0,5 \%$ de la población total del país y con una densidad menor a 0,1 habitante por $\mathrm{km}^{2}$.

\footnotetext{
50 Sin contar el Territorio de Magallanes, virtualmente despoblado, la superficie de Chile en 1907 correspondía a $585.824 \mathrm{~km}^{2}$. En ese momento, las provincias de Tacna, Tarapacá, Antofagasta, Malleco y Cautín, cuyos territorios se incorporaron en la década de 1880 representaban $214.439 \mathrm{~km}^{2}$, equivalentes al $37 \%$ del total. Censo de Población (1907).

51 Cariola \& Sunkel (1982), p. 80.
} 
Se observa que la incorporación efectiva de los territorios que luego conformarían las provincias de Antofagasta, Tacna, Tarapacá, Malleco y Cautín en la primera mitad de la década de 1880 amplió la disponibilidad de recursos naturales por habitante. Si bien entre 1875 y 1885 la población crece en forma importante -ya que las nuevas regiones no eran territorios vacíos-, más lo hace la disponibilidad de tierra. Como consecuencia ésta se hizo más abundante en relación al trabajo. La mayor disponibilidad de tierra por trabajador comenzaría a revertirse luego como resultado del crecimiento demográfico, agotándose hacia la mitad de la primera década del siglo xx. En 1907 la dotación relativa de factores es similar a la de 1875 .

La incorporación de estos territorios a la economía chilena causó un proceso de migraciones internas que supuso una reasignación de la mano de obra que emigró hacia las nuevas regiones.

El Cuadro 8 permite apreciar las diferentes tasas de crecimiento demográfico según las regiones. La región del Norte Grande — compuesta por las ex provincias peruanas de Tacna y Tarapacá y la antigua provincia boliviana de Antofagastamuestra un salto demográfico entre 1875 y 1885. Su población pasa de 2.017 a 87.950 habitantes, lo que supone un ritmo de crecimiento anual del $48,9 \%$. Luego sigue creciendo en el entorno del $4 \%$ anual hasta 1907, año en que representa el $8 \%$ de la población total. En los territorios incorporados al sur del Valle Central como resultado de la derrota de los araucanos, la población de los departamentos que desde 1887 conformarían las provincias de Malleco y Cautín pasaría, entre 1875 y 1885, de 20.056 a 59.472 habitantes en el primer caso y de 6.446 a 38.141 en el segundo, lo que supone en conjunto una tasa de crecimiento anual del 13,9\%. A partir de 1885 ambas provincias continúan creciendo a un ritmo muy superior al promedio nacional. Como resultado pasan de representar el 1\% del total en 1875 al 10\% en 1907 (Cuadro 5).

En contrapartida, las regiones de poblamiento tradicional muestran escaso dinamismo en su crecimiento poblacional, e incluso tasas negativas, como la región del Norte Chico y Concepción entre 1885 y 1895. En el caso de la Zona Central, si bien muestra un crecimiento leve, del entorno del 1\% anual -inferior de todos modos al promedio nacional- el mismo esconde dos realidades diferentes. Por una parte, la expansión de la población urbana, que al influjo de la expansión de Santiago crece a un ritmo en el entorno del 2\% anual. La población rural del Valle Central en cambio, decrece a una tasa del -0,40\% anual entre 1885 y 1895, a la vez que su participación en el total cae, entre 1875 y 1907, del 68\% al 54\% (Cuadro 8$)^{53}$.

\footnotetext{
$52 \quad$ Hurtado (1966), cuadro 2.

53 Hurtado (1966), cuadro 2.
} 


\section{CUADRO 8}

EVOLUCIÓN DE LA POBLACIÓN POR REGIONES. PARTICIPACIÓN EN EL TOTAL DE HABITANTES Y TASAS DE CRECIMIENTO PARA PERÍODOS INTERCENSALES

\begin{tabular}{lcrrrrrr}
\hline & \multicolumn{3}{c}{$\begin{array}{c}\text { Población regional en relación } \\
\text { al total para años censales (\%) }\end{array}$} & \multicolumn{3}{c}{$\begin{array}{c}\text { Tasa de crecimiento de la población } \\
\text { por regiones entre censos }\end{array}$} \\
\cline { 2 - 8 } & $\mathbf{1 8 7 5}$ & $\mathbf{1 8 8 5}$ & $\mathbf{1 8 9 5}$ & $\mathbf{1 9 0 7}$ & $\mathbf{1 8 7 5 - 1 8 8 5}$ & $\mathbf{1 8 8 5 - 1 8 9 5}$ & $\mathbf{1 8 9 5 - 1 9 0 7}$ \\
\hline Norte Grande & 0,1 & 4 & 6 & 8 & $48,9 \%$ & $3,9 \%$ & $4,0 \%$ \\
Norte Chico & 11 & 10 & 8 & 7 & $0,6 \%$ & $-0,9 \%$ & $0,7 \%$ \\
Zona Central & 68 & 61 & 60 & 54 & $0,9 \%$ & $0,9 \%$ & $0,5 \%$ \\
Concepción & 13 & 14 & 12 & 11 & $2,8 \%$ & $-0,5 \%$ & $0,9 \%$ \\
Frontera & 0,9 & 4 & 6 & 10 & $13,9 \%$ & $6,1 \%$ & $5,9 \%$ \\
Los Lagos & 4 & 5 & 5 & 7 & $3,1 \%$ & $2,0 \%$ & $4,0 \%$ \\
Austral & 3 & 3 & 3 & 3 & $1,3 \%$ & $0,6 \%$ & $1,1 \%$ \\
\hline Total & 100 & 100 & 100 & 100 & $2,0 \%$ & $1,0 \%$ & $1,5 \%$ \\
\hline
\end{tabular}

Fuente: Elaborado a partir del Censo de Población de 1907, excepto la información para el Norte Grande en 1875 que se toma de Hurtado (1966 cuadro 2). El análisis por provincia del Censo informa de la evolución desde 1865 de la población de cada departamento que integra la provincia en 1907. De esta forma es posible reconstruir la población de provincias para un período anterior a que éstas se hayan conformado, como por ejemplo la de la provincia de Malleco antes de 1887, año de su creación. La región del Norte Grande comprende las provincias de Tacna, Tarapacá, y Antofagasta. La del Norte Chico las de Atacama y Coquimbo. La Zona Central las de Aconcagua, Valparaíso, Santiago, O’Higgins, Colchagua, Curicó, Talca, Linares, Maule, y Ñuble. La región de Concepción agrupa a las provincias de Concepción, Arauco y Bío-Bío. La región Frontera las de Malleco y Cautín. Los Lagos corresponde a Valdivia y Llanquihue, y la zona Austral a las provincias de Chiloé y al Territorio de Magallanes.

Es de destacar por tanto, que si bien la disponibilidad de tierra por habitante comienza a caer luego de 1885, lo hace desde un nivel muy diferente al de 1875; este último se alcanzaría recién a comienzos del siglo xx. Pero a su vez, los movimientos demográficos del período 1885-1895 hacen que mientras la disponibilidad de tierra cae a nivel nacional, ésta se incremente en la zona rural de la región central.

La incorporación de nuevas tierras y los movimientos demográficos que hemos descrito condujeron a una transformación importante de la geografía productiva chilena. El concepto de "expansión de la frontera" ha sido utilizado para analizar estas transformaciones, tanto el auge salitrero al norte, como la ocupación de las tierras agrícolas al sur ${ }^{54}$.

El desarrollo de la minería del salitre en el Norte Grande supuso mucho más que una fuente abundante de divisas para la economía chilena. La minería nortina propició una serie de transformaciones a diversos niveles. Además de aumentar su participación en el producto, la minería fue el primer sector capitalista de Chile, 
debido a su escala de producción, las tecnologías utilizadas, y el tipo de mercado laboral centrado en la relación salarial del que nacería el movimiento obrero ${ }^{55}$.

Si bien desde antes de la guerra existían vínculos económicos entre las regiones salitreras de Perú y Bolivia y Chile, su importancia para éste último era mínima comparada con lo ocurrido luego de 1880. Por otra parte, la participación chilena en los orígenes de la industria salitrera no habría sido tan excluyente e importante como la perspectiva clásica ha sostenido ${ }^{56}$. En 1879 los capitales chilenos invertidos en la explotación del salitre representaban poco más que los británicos, siendo un $19 \%$ del total los primeros y un 13,5\% los segundos, en el marco de una industria controlada por capitales peruanos ${ }^{57}$. A su vez las empresas europeas eran más eficientes que la chilenas debido a su mejor acceso al crédito y su superioridad administrativa, técnica y comercial ${ }^{58}$, y por ello fueron menos afectadas por la crisis de la década de $1870^{59}$.

Asimismo, fue luego de la guerra que se introdujo el sistema Shanks, tecnología clave de la explotación salitrera durante todo su período de auge. Éste posibilitó la explotación de caliches de menor ley, a la vez que permitía ahorrar en combustible y mano de obra. Como resultado de su introducción, la capacidad productiva se triplicó a lo largo de la década de 1880, creciendo no sólo la producción sino la productividad. A su vez los costos se reducían en un $40 \%$.

La exitosa y rápida ocupación militar de la provincia boliviana de Antofagasta en febrero de 1879, permitió la casi inmediata explotación por parte de Chile de sus riquezas naturales. Así, en dos años la producción chilena de salitre se multiplicó por 304, pasando de 741.470 kilogramos en 1878, a 226.090.115 en $1880^{61}$. Esta explosión productiva requirió el concurso de ingentes cantidades de mano de obra, lo que provocó un importante crecimiento de la población nortina. La totalidad de los trabajadores del salitre recibían un salario a cambio de su trabajo, y éstos eran más altos, del entorno del doble o triple de los ofrecidos en Chile central ${ }^{62}$. Aunque el mayor costo de vida y el pago en fichas reducían esta brecha, la misma era suficientemente significativa para que miles de trabajadores se dirigiera hacia allí a pesar de las condiciones físicas y climáticas inhóspitas ${ }^{63}$.

En el Sur, la otra zona de expansión territorial, la incorporación de nuevos territorios se produjo en dos etapas. En la década de 1860 se avanzó hasta el Malleco

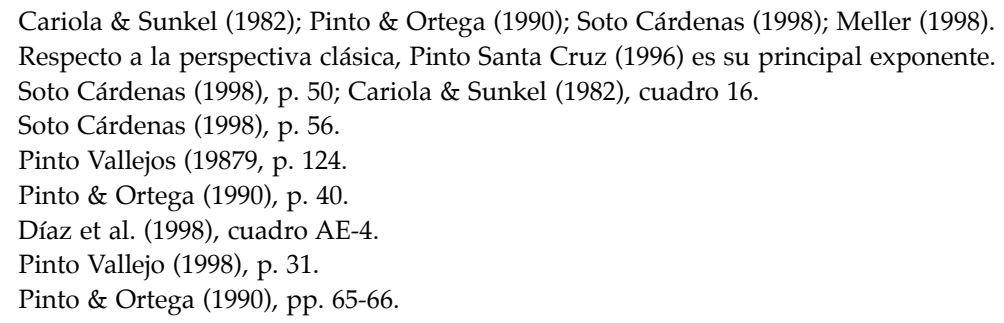


y fue dominada la región costera del río Toltén, "pero sólo en 1882 se aplastó definitivamente la resistencia organizada" ${ }^{64}$. Así, el territorio central de Chile se unía con los territorios australes que, desde mediados de siglo, eran habitados por colonias de inmigrantes. También este proceso se ha caracterizado como de expansión de la frontera, por sus similitudes con el caso norteamericano ${ }^{65}$.

La nueva región se incorporó rápidamente a la producción de bienes agrícolas. Si a fines de la década de 1870 las futuras provincias de Malleco y Cautín —entonces parte del territorio de colonización de Angol— no producen trigo, en 1884 / 85 explican el 8\% de la producción total, porción que asciende al 22\% en 1904/05

El proceso de colonización fue el resultado de la combinación de tres vertientes: dos estrategias públicas y un proceso espontáneo. En cuanto a las primeras, el Estado se debatió entre dos alternativas, "una defendía la colonización por parte de extranjeros y chilenos que trabajasen su propia tierra", por lo que se entregaron tierras a oficiales y tropa del ejército desmovilizado, y "mercedes" a los indígenas. La segunda alternativa era defendida por quienes sostenían que el Estado debía abstenerse de intervenir dejando "en libertad a los particulares para que compraran lo que les pareciera" ${ }^{\prime 67}$. Pero también, y en parte debido a la incertidumbre institucional generada por el conflicto entre las distintas estrategias públicas, se instalaron numerosos "colonos espontáneos", animados por la promesa de tierras. El resultado fue una estructura de propiedad heterogénea, origen de múltiples conflictos posteriores ${ }^{68}$.

En resumen, las victorias militares de principios de la década de 1880 modificaron sustancialmente la geografía económica del Chile. En el Norte la incorporación de los territorios salitreros supuso la transformación tanto del país que los anexionó, como de las técnicas productivas utilizadas en la actividad minera. Para Chile supuso la formación de su primer sector plenamente capitalista, caracterizado por importantes concentraciones de trabajadores atraídos por salarios hasta tres veces más altos que en la región central. Para la actividad salitrera supuso un conjunto de cambios productivos y de escala que, al menos durante nuestro período, la ubicaron en la frontera tecnológica. En el Sur, los trabajadores provenientes del Valle Central incorporaron rápidamente la nueva región a la economía productiva, trasformándola en el nuevo centro de la producción cerealera. Las zonas rurales de la región central alimentaron los procesos migratorios y perdieron población, lo que redujo la oferta de trabajo rural en las zonas de poblamiento tradicional. Como consecuencia, luego de 1880 el trabajo se hizo más escaso, en particular en las zonas rurales tradicionales.

\footnotetext{
$64 \quad$ Hurtado (1966), p. 68.

65 Hurtado (1966), p. 68.

66 Cariola \& Sunkel (1982), p. 185.

67 Bengoa (1988), pp. 251-252.

68 Bengoa (1988), p. 253.
} 


\subsection{Impacto de la expansión de la frontera en la desigualdad}

Las consecuencias para la desigualdad de la expansión de la frontera, se observan a nivel regional y sectorial. En cuanto a la evolución regional, el Cuadro 9 muestra que entre 1875 y 1885 la desigualdad cae en los extremos que incorporan nuevas regiones al proceso productivo chileno, particularmente en el Norte, donde el cambio en la dotación de factores es más rápido y drástico.

La evolución de la desigualdad regional tiene un correlato en la sectorial. El Cuadro 10 muestra que entre 1875 y 1885 la desigualdad cae en la minería.

CUADRO 9

EVOLUCIÓN DE LA DESIGUALDAD REGIONAL EN 1875, 1885 Y 1907

\begin{tabular}{clccc}
\hline Año & \multicolumn{1}{c}{ Región } & GE (0) & GE (1) & GINI \\
\hline \multirow{2}{*}{1875} & Norte (Chico) & 0,88 & 2,13 & 0,68 \\
& Centro & 0,43 & 0,79 & 0,50 \\
& Sur & 0,35 & 0,65 & 0,43 \\
\hline \multirow{2}{*}{$\mathbf{1 8 8 5}$} & Norte (Chico y Grande) & 0,46 & 0,78 & 0,51 \\
& Centro & 0,44 & 0,70 & 0,50 \\
& Sur & 0,29 & 0,51 & 0,39 \\
\hline \multirow{2}{*}{$\mathbf{1 9 0 7}$} & Norte(Chico y Grande) & 0,43 & 0,80 & 0,49 \\
& Centro & 0,41 & 0,72 & 0,48 \\
& Sur & 0,38 & 0,82 & 0,44 \\
\hline
\end{tabular}

Fuente: Estimación propia a partir de bases de datos elaborada para años puntuales o mojones. Calculado a partir de estimación del Ingreso Nacional Bruto.

CUADRO 10

EVOLUCIÓN DE LA DESIGUALDAD SECTORIAL EN 1875, 1885 Y 1907

\begin{tabular}{clccc}
\hline Año & Sector & GE (0) & GE (1) & GINI \\
\hline \multirow{3}{*}{1875} & Agro & 0,32 & 0,59 & 0,39 \\
& Minería & 1,52 & 2,66 & 0,81 \\
& Industria & 0,41 & 0,58 & 0,48 \\
& Servicios & 0,42 & 0,68 & 0,47 \\
\hline \multirow{3}{*}{1885} & Agro & 0,28 & 0,61 & 0,35 \\
& Minería & 0,75 & 1,32 & 0,60 \\
& Industria & 0,29 & 0,48 & 0,40 \\
& Servicios & 0,35 & 0,51 & 0,45 \\
\hline \multirow{3}{*}{1907} & Agro & 0,29 & 0,88 & 0,34 \\
& Minería & 0,67 & 1,32 & 0,54 \\
& Industria & 0,21 & 0,27 & 0,34 \\
& Servicios & 0,59 & 0,70 & 0,57 \\
\hline
\end{tabular}

Fuente: Estimación propia a partir de bases de datos elaborada para años puntuales o mojones. Calculado a partir de estimación del Ingreso Nacional Bruto. 
El análisis del Gráfico 3 permite apreciar que las consecuencias de la expansión de la frontera sobre la desigualdad se produjeron siguiendo dos etapas. En un primer momento se incorporan en forma abrupta nuevas tierras al norte, lo que cambia en forma drástica y rápida la estructura de la dotación de factores de esa región. El Gráfico 3 muestra que la caída en la porción de las utilidades respecto del total - cerca de quince puntos entre 1880 y 1885- coincide con la caída de la desigualdad en la región del Norte y el sector minero entre 1875 y 1885- según muestran los índices de Gini y entropía calculados a partir de las bases de datos de esos años ${ }^{69}$.

\section{GRÁFICO 3}

PORCENTAJE DEL INGRESO TOTAL CAPTADO POR LAS UTILIDADES EN LOS SECTORES

MINERO Y AGRÍCOLA (1880-1905)

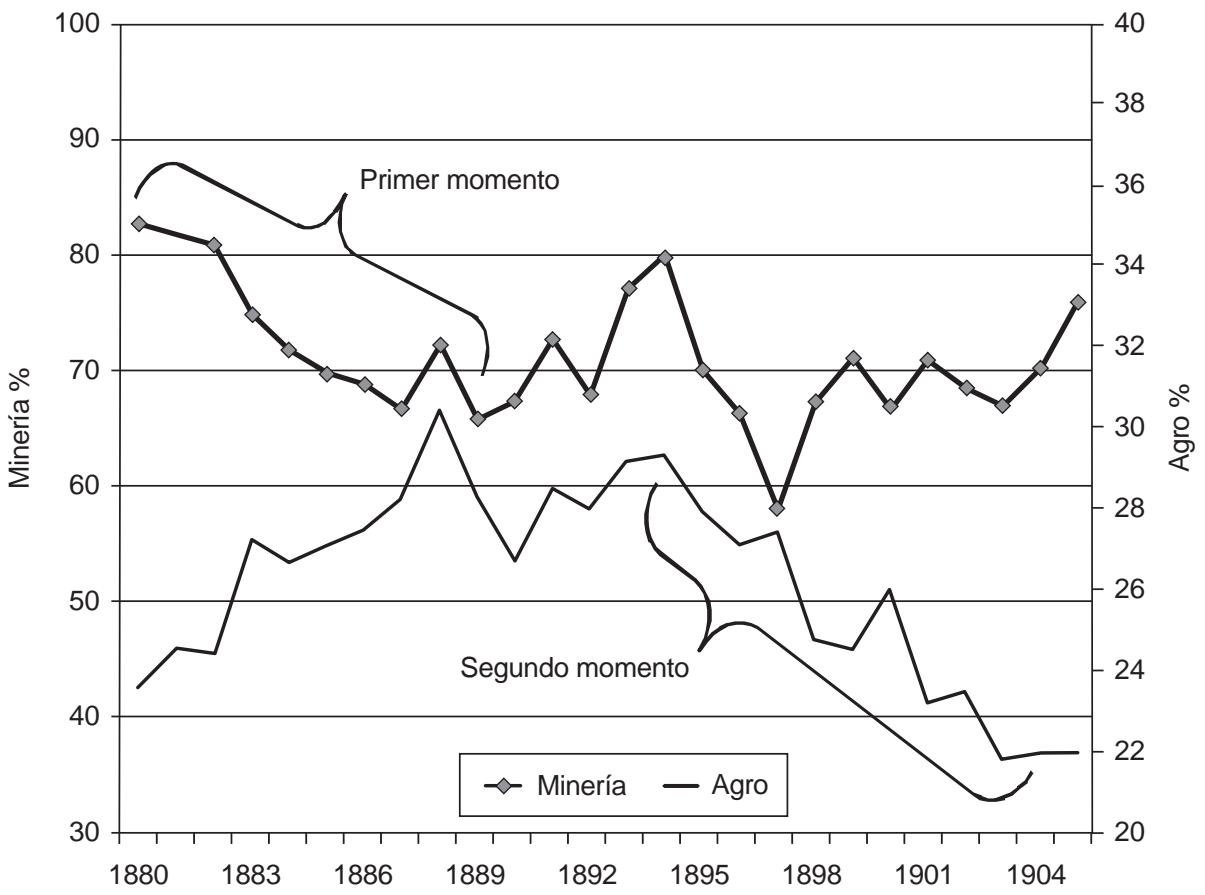

Fuente: Estimación propia. Sobre los distintos "momentos" ver texto. Calculado a partir de estimación del Ingreso Interior Bruto

69 Si bien las estimaciones del índice de Gini no tienen en cuenta la porción de utilidades del salitre enviada al extranjero, el porcentaje de las utilidades en el total del ingreso que se muestra en el Gráfico 3 sí lo hace. En todo caso, ambos indicadores muestran una caída de la desigualdad en el sector de la minería entre $1875 / 80$ y 1885. 
Sin embargo, los altos salarios atraen a gran cantidad de trabajadores y la desigualdad en el Norte y la minería deja de caer. Hacia 1890 y, más allá de los casos excepcionales de los años 1893 y 1894 —donde la porción de las utilidades trepó hasta el entorno del 80\% - y del año 1897 — cuando cayó por debajo del 60\% — la participación de las utilidades en el total se mantiene en el entorno de $70 \%$ por lo que resta del período. Con ello culmina el Primer momento en la caída de la desigualdad.

El segundo momento ocurre una vez que la emigración de la fuerza de trabajo hacia la frontera modifica la dotación relativa de factores en la zona central y el sector agrícola. La porción que los terratenientes captaba del ingreso agrario cayó entonces del entorno del 28\% hacia 1895, hasta el $20 \%$ una década después.

La expansión de la frontera supuso que tanto al norte como al sur del Valle Central se abrieran nuevas oportunidades para la población de gañanes trashumantes. Estos se dirigieron al Norte en busca de salarios altos, al Sur esperanzados por la expectativa de tierras, y a las ciudades, que el crecimiento minero alimentaba. La emigración de los trabajadores de la zona central, que alcanzara su punto máximo en algún momento cercano a 1895, alteró la relación tierra/trabajo, haciendo a éste más escaso, lo que incidió en su retribución.

Los gañanes, aquellos "asalariados de la agricultura" proporcionaban mano de obra estacional a la Hacienda:

"Estos trabajadores trashumantes, conocidos en la historia agraria chilena como 'torrantes', como 'afuerinos' permanentes, 'linyeras', 'andantes' se iban trasladando de faena en faena de acuerdo a los tiempos. (...) Recibían generalmente una ración de comida, la galleta campesina, y el resto de su salario en dinero metálico. Eran los asalariados de la agricultura"70.

Su emigración de las zonas rurales del Valle Central presionó al alza sus salarios, que comienzan a crecer cuando ellos son más escasos (Gráfico 4$)^{71}$.

Entre 1894 y 1905, el ingreso de los gañanes pasó de representar el 25\% al 48\% del ingreso medio ${ }^{72}$. En los mismos años, el ingreso de los terratenientes pasa de equivaler 258 a 181 veces el de los gañanes (Gráfico 5).

Bengoa (1988), p. 119.

Las mayores oportunidades que suponía para los trabajadores de la región central la incorporación de nuevas tierras ya habían sido destacadas por Pinto Santa Cruz (1996), p. 132.

72

Rodríguez Weber (2009), cuadro AE-9. 


\section{GRÁFICO 4}

OFERTA DE TRABAJO AGRÍCOLA E INGRESO REAL DE TRABAJADORES RURALES (1885-1905)

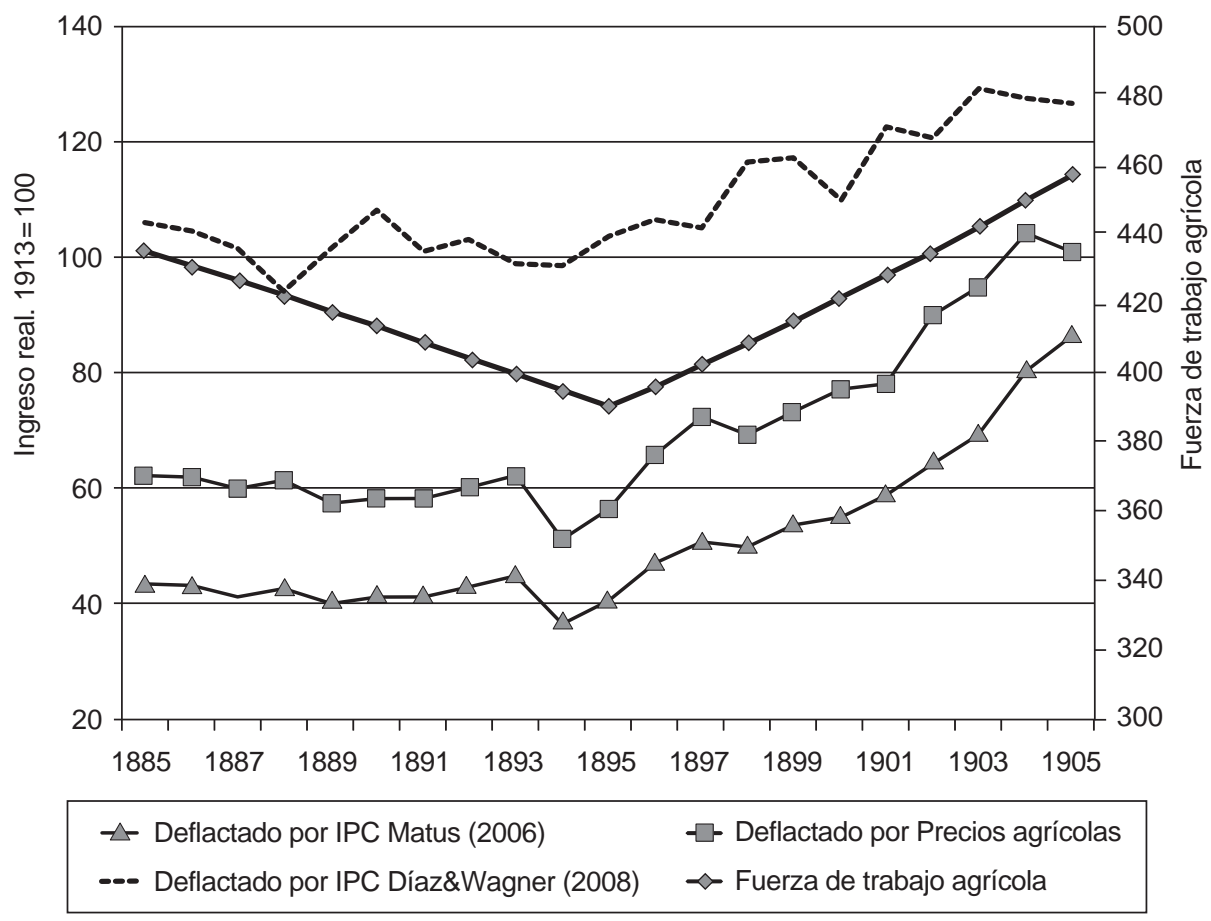

Fuente: El ingreso de los gañanes se obtuvo de Bauer (1994; Cuadro 31 pág. 182) anualizados según días trabajados tomados de Wagner (1992) Se ha deflactado por Índice de Precios Agrícolas IPALS (Wagner 1992), y por dos Índices de Precios al Consumo (IPC) tomados de Díaz \& Wagner (2008) y Matus (2006). La fuerza de trabajo agrícola es de Braun et al (2000). 


\section{GRÁFICO 5}

RELACIÓN ENTRE EL INGRESO DE LOS TERRATENIENTES 1 RESPECTO DEL DE GAÑANES

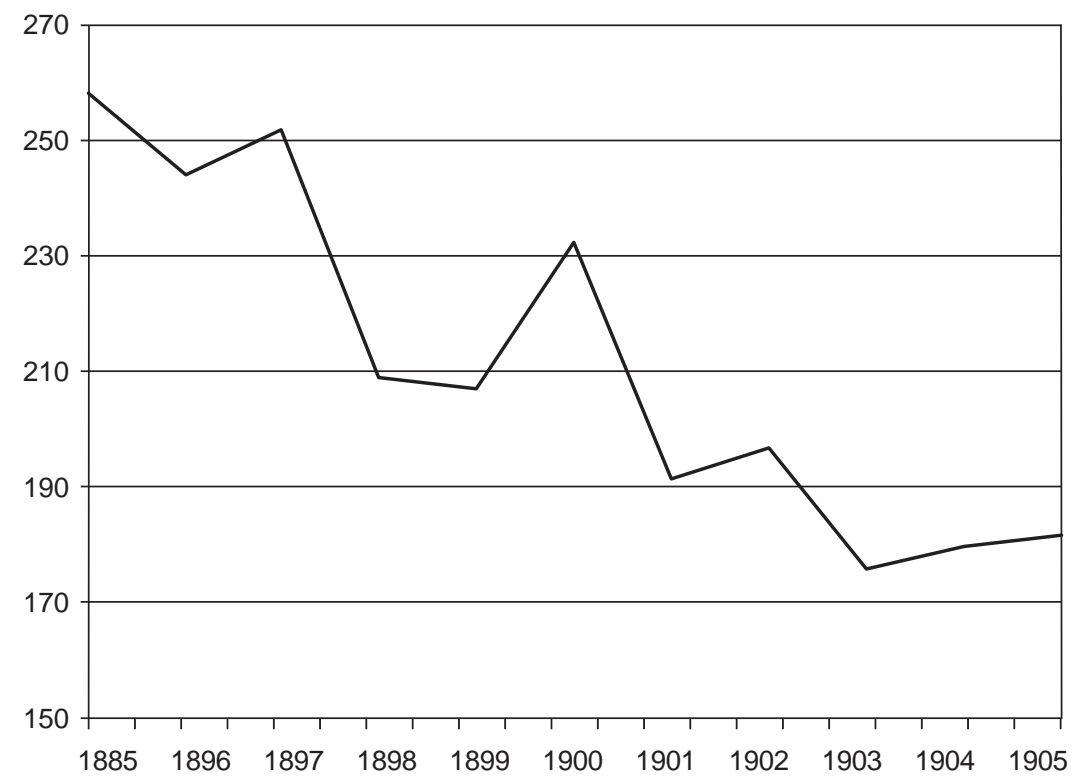

Fuente: Estimación propia. La categoría Terratenientes 1 incluye a los propietarios con mayores ingresos.

Con el objetivo de estimar el impacto que la mejora en el ingreso de los gañanes produjo en la evolución global de la desigualdad realizamos un ejercicio contrafactual suponiendo que la evolución de su ingreso nominal a partir del año 1896 se corresponde con la evolución del índice de precios agrícolas IPALS ${ }^{73}$. Es decir, el ingreso de gañanes se supone constante en términos de precios agrícolas - los que mostraron una importante tendencia al alza en el período-. Debe tenerse en cuenta que en el ejercicio el ingreso perdido por los gañanes no se asigna a ningún otro sector. Si supusiéramos que la ausencia de incremento del salario real de los gañanes hubiera beneficiado a los terratenientes - lo que es razonable-, la diferencia entre el ejercicio contrafactual y la estimación original sería más importante. Por lo anterior puede considerarse que esta diferencia - que muestra el Gráfico 6- constituye la hipótesis de minima respecto del impacto que el incremento del salario real de los gañanes tuvo en la desigualdad global.

En síntesis, la expansión de la frontera condujo a una serie de transformaciones que se produjeron en dos etapas, lo que explica la caída en dos fases de los índices de desigualdad personal (Gráficos 2 y 3). 


\section{GRÁFICO 6}

EJERCICIO CONTRAFACTUAL. ESTIMACIÓN DEL ÍNDICE DE GINI SUPONIENDO QUE NO SE PRODUJERA INCREMENTO EN EL INGRESO REAL DE GAÑANES (1895-1909)

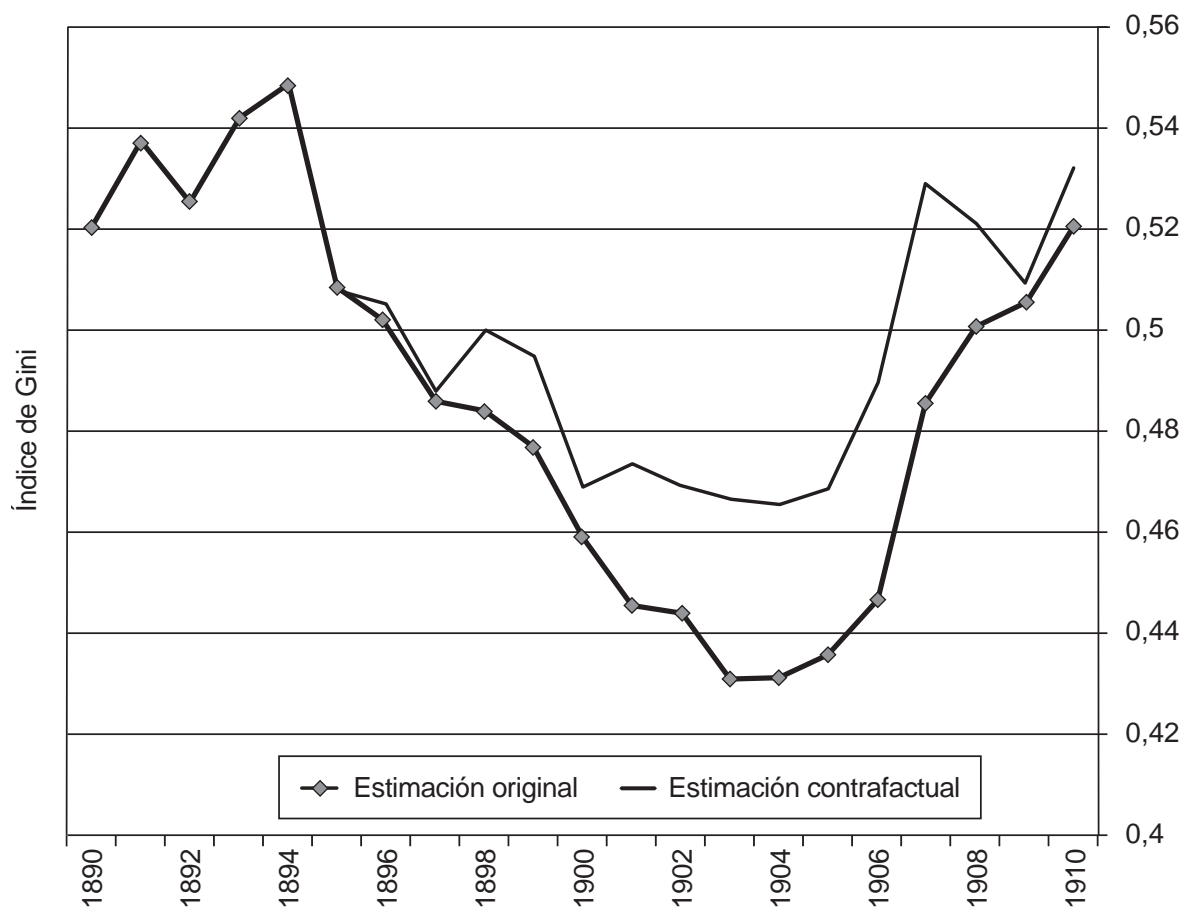

Fuente: Elaboración propia. Para el ejercicio contrafactual se ha supuesto que el ingreso de los gañanes posterior al año 1896 evoluciona según el Índice de Precios Agrícolas (IPALS) de Wagner (1992). Al mantener constantes en términos de precios agrícolas el ingreso de los gañanes, el ejercicio muestra el límite inferior del impacto que su aumento tuvo en la caída de la desigualdad. El mismo habría sido superior si los salarios de gañanes evolucionaran en forma lineal entre 1894 y 1913, o si el ingreso perdido en el ejercicio se asignara a los terratenientes

En la primera mitad de la década de 1880, el crecimiento de la cantidad relativa de tierra condujo a una reducción de la desigualdad en la minería y en la región del Norte. A partir de la década de 1890, la emigración hacia las zonas de reciente incorporación redujo la oferta de trabajo rural, lo que provocó el aumento en el ingreso real de los peones-gañanes del Valle Central, y la reducción de la desigualdad en la distribución del Ingreso Nacional Bruto medida por los índices de desigualdad personal — Gini, Theil y GE (0)— entre 1895 y $1905^{74}$.

74 Señalar que el cambio en la dotación de factores y el incremento en el ingreso de los gañanes condujeron a la reducción de la desigualdad en la distribución del ingreso no supone afirmar que fueron los únicos factores actuantes en este sentido. En Rodríguez Weber (2009) y Bértola \& Rodríguez Weber (2009) se presenta una visión más comprehensiva del período. 


\subsection{Agotamiento del efecto frontera en la desigualdad}

La caída de la desigualdad causada por las transformaciones asociadas a la expansión de la frontera resultó ser un fenómeno pasajero. Dicha tendencia se revirtió hacia 1905, cuando la distribución del ingreso se deterioró, alcanzando en los años siguientes niveles más altos aún que los existentes antes de $1880^{75}$. Ello se explica, por una parte, por el carácter intrínsecamente coyuntural del proceso de expansión de la frontera. En el caso de Chile, el mismo se agota hacia 1907 cuando la relación tierra/trabajo es similar a la de 1875 (Cuadro 7), y la población rural del Valle Central alcanza niveles similares a los del año $1885^{76}$.

Pero más importante para explicar esta reversión es que, junto con lo anterior, se observa una conjunción de factores institucionales que favorecieron la reversión de la tendencia igualitaria analizada en este trabajo. Ello se aprecia en especial en la forma en que se distribuyeron las tierras del Sur. Entre las diversas alternativas posibles el Estado adoptó una que permitió a la antigua Hacienda de origen colonial, "colonizar" el Sur, imprimiendo allí su sello, caracterizado por el latifundio y la desigualdad ${ }^{77}$. Asimismo, la estrategia seguida para enfrentar los problemas del sector minero condujo, junto a otras razones, a un fuerte proceso inflacionario que, a partir de la primera década del siglo XX, afectó en primer lugar a los asalariados. Cuando éstos intentaron enfrentar el deterioro de sus ingresos reales, el Estado les respondió con las masacres obreras perpetradas en la pampa salitrera ${ }^{78}$. Como consecuencia de las mismas, el naciente movimiento obrero fue incapaz de impedir que el alza de precios deteriorara su ingreso real, conduciendo así a un aumento de la desigualdad. La expansión de la frontera supuso una oportunidad para lograr el crecimiento económico con mayor equidad. Sin embargo, una vez que agotó sus efectos, se hicieron patentes características de la economía y el entramado socioinstitucional chileno tradicional que echaron por tierra dicha oportunidad ${ }^{79}$.

\section{Conclusiones}

La crisis del primer ciclo exportador, y los acontecimientos que la siguieron, significaron un quiebre en la historia de Chile. El país amplió notablemente su territorio como resultado de dos victorias militares, una al norte sobre dos países vecinos, y otra al sur, sobre la población aborigen.

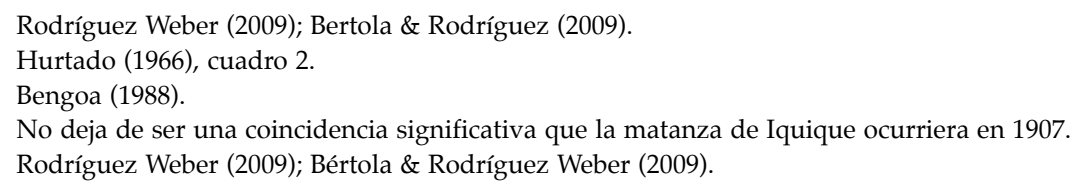


La ampliación del territorio promovió un conjunto de transformaciones en la formación social y económica chilena. En primer lugar, ella modificó la dotación de factores al aumentar la abundancia relativa de tierra. En segundo lugar afectó la composición de la fuerza de trabajo. En el Norte se generó un mercado de trabajo capitalista con salarios que al ser promediamente más altos que en el resto del país, atraían mano de obra. En el Sur, y mientras se definía el modelo de ocupación, se generó la expectativa de acceso a la tierra. A lo anterior se agrega la posibilidad de encontrar trabajo en las crecientes urbes, y en la construcción de ferrocarriles, ambos procesos alimentados por la riqueza salitrera. La población trashumante de los gañanes vio así aumentadas sus posibilidades de emigración, tradicionalmente reducidas al cambio de una hacienda a otra, o al cruce de fronteras.

El primer impacto de la ampliación de la dotación del factor tierra fue, a la vez que una profundización de la especialización en recursos naturales, un deterioro de la remuneración al mismo en relación al factor ahora más escaso —el trabajo-. Ello resultó en una caída de la desigualdad que se verificó en dos etapas. En un primer momento, la desigualdad cayó en la región más rápidamente incorporada a la economía de Chile — el Norte- y en el sector a ella asociada - la minería-. Allí, mientras se desarrollaba el primer sector plenamente capitalista del país, los altos salarios atraían a trabajadores de las zonas tradicionales. Como resultado se originó una corriente migratoria desde las zonas rurales tradicionales - Norte Chico y Valle Central- hacia los territorios de reciente incorporación. El proceso migratorio condujo a la segunda etapa en la caída de la desigualdad, cuando la reducción de la oferta de trabajo en el medio rural de la región central provocó un aumento de los ingresos de los peones.

La falta de evidencia histórica sólida, aunada a la centralidad del modelo $\mathrm{H}$ O-S-S en los estudios sobre la desigualdad durante el período ha obstaculizado, hasta ahora, la valoración adecuada del impacto que la expansión de la frontera tuvo sobre la distribución del ingreso en Chile. La evidencia presentada para el caso chileno, junto a procesos similares señalados para otros países — como el caso de Australia-, apunta a la necesidad de mayor investigación empírica y mejor teorización para comprender los vínculos que un proceso complejo como la primera globalización tuvo con la desigualdad en los países exportadores de bienes primarios.

\section{Bibliografía}

ÁLVAREZ, Jorge; BÉRTOLA, Luis \& PORCILE Gabriel (2007) (eds.): Primos Ricos y Empobrecidos: Crecimiento, Distribución del Ingreso e Instituciones en Australia-Nueva Zelanda vs Argentina-Uruguay, Montevideo, Ediciones Fin de Siglo. 
BAUER, Arnold (1970): “Expansión económica en una sociedad tradicional. Chile Central en el siglo XIX", en Historia, Santiago de Chile, Universidad Católica, Instituto de Historia, n. ${ }^{\circ}$ 9, pp. 137-235.

-(1994): La sociedad rural chilena. Desde la conquista española a nuestros días, Santiago de Chile, Andrés Bello.

BENGOA, José (1988): Historia social de la Agricultura Chilena. Tomo I. El poder y la subordinación, Santiago de Chile, Ed. Sur.

-(1990): Haciendas y campesinos. Historia social de la Agricultura Chilena. Tomo II, Santiago de Chile, Ed. Sur.

BERTOLA, Luis (2005): “A 50 años de la Curva de Kuznets: Crecimiento y distribución del ingreso en Uruguay y otras economías de nuevo asentamiento desde 1870", Investigaciones de Historia Económica, 3/2005, pp. 135-176.

BERTOLA, Luis \& WILLIAMSON, Jeffrey (2006): "Globalization in Latin America before 1940", en BULMER-THOMAS, Coatsworth \& CORTÉS CONDE (ed.), Cambridge Economic History of Latin America, vol. II, pp. 11-56.

BERTOLA, Luis \& RODRÍGUEZ WEBER, Javier (2009): "Between La Longue Durée, Globalization and the Expansion of the Frontier: Income Inequality in Chile 1860-1930", paper presented at the Conference A Comparative Approach to Inequality and Development: Latin America and Europe, Madrid, mayo 2009.

BERTOLA, Luis; CASTELNOVO, Cecilia; RODRÍGUEZ, Javier \& WILLEBALD, Henry (2010): "Between the colonial Heritage and the first globalization boom: on income inequality in the South Cone", en Revista de Historia Económica, Journal of lberian and Latin American Economic History, vol. 28, n. ${ }^{\circ}$ 2, pp. 307-341.

BRAUN, Juan; BRAUN, Matías; BRIONES, Ignacio; DÍAZ, José; LÜDERS, Rolf \& WAGNER, Gert (2000): “Economía Chilena 1810-1995: Estadísticas históricas", Santiago de Chile, Instituto de Economía - Pontificia Universidad Católica, Documento de Trabajo . $^{\circ} 187$.

CARIOLA, Carmen \& SUNKEL, Octavio, (1982): Un siglo de Historia económica de Chile 1830-1930. Dos ensayos y una bibliografía, Madrid, Ediciones Cultura Hispánica.

COMISIÓN CENTRAL DEL CENSO (1907): Censo 1907. Memoria presentada al Supremo Gobierno.

DÍAZ, José; LÜDERS, Rolf \& WAGNER, Gert (1998): “Economía chilena 1810-1995. Evolución cuantitativa del producto total y sectorial", Santiago de Chile, Instituto de Economía - Pontificia Universidad Católica, Documento de Trabajo n. ${ }^{\circ}$ 186.

—(2007): “Chile 1810-2000. La República en cifras (en preparación)”.

DÍAZ, José \& WAGNER, Gert (2008): "Inflación y tipo de cambio: Chile1810-2005", Santiago de Chile, Instituto de Economía - Pontificia Universidad Católica, Documento de Trabajo n. ${ }^{\circ} 328$. 
EMERY, J. C. Herbert; INWOOD, Kris \& THILLE, Henry (2007): “Hecksher-Ohlin in Canada: new estimates of regional wages and land prices", en Australian Economic History Review, vol. 47, n. ${ }^{\circ} 1$, pp. 22-48.

GÁLVEZ, Thelma \& BRAVO, Rosa (1992): “Siete décadas de registro del trabajo femenino", en Estadística \& Economía, Santiago, n. ${ }^{\circ}$ 5, diciembre, pp. 2-52

GREASLEY, David; INWOOD, Kris \& SINGLETON, John (2007): “Factor prices and income distribution in less industrialised economies 1870-1939", en Australian Economic History Review, vol. 47, n. ${ }^{\circ}$ 1, pp. 1-5.

HARLEY, Kcnick (2007): “Comments on factor prices and income distribution in less industrialised economies 1870-1939: refocusing on the frontier", en Australian Economic History Review, vol. 47, n. ${ }^{\circ}$ 3, pp. 238-248.

HUTCHISON, Elizabeth (2000): “La historia detrás de las cifras: La evolución del censo chileno y la representación del trabajo femenino, 1895-1930", en Historia n. ${ }^{\circ} 33$, pp. 417-434.

MATUS, Mario (2006): “Genealogía de los Precios Inflacionarios en Chile: Dinámicas de Precios Durante el Ciclo Salitrero 1880-1930", Tesis Magíster, Universidad de Chile, en línea http:/ / www.cybertesis.cl/tesis/uchile/2006/matus_m/sources/matus_m.pdf.

MELLER, Patricio, (1998): “Un siglo de economía política chilena. (1890-1990)”, Ed. Andrés Bello, Santiago de Chile.

O'ROURKE, Kevin \& WILLIAMSON, Jeffrey (1999): Globalization and history: The evolution of late nineteenth century economy, Cambridge, MIT Press.

ORTIZ LETELIER, Fernando (2005): El movimiento obrero en Chile. (1891-1919), Santiago de Chile, Lom ediciones.

PINTO SANTA-CRUZ, Aníbal (1996): Chile, un caso de desarrollo frustrado, Santiago de Chile, Universidad de Santiago.

PINTO VALLEJO, Julio (1998): Trabajos y rebeldías en la pampa salitrera, Santiago de Chile, Universidad de Santiago de Chile.

PINTO VALLEJO, Julio \& ORTEGA MARTÍNEZ, Luis (1990): Expansión minera y desarrollo industrial. Un caso crecimiento asociado, Santiago de Chile, Universidad de Santiago de Chile.

PRADOS, Leandro (2005): "Growth, inequality and poverty in Latin America. Historical evidence, controlled conjectures", Universidad Carlos III. Working Paper 05-41(04), http:/ / www.uc3m.es/uc3m/dpto/HISEC/Doctrab/ 2005/ wp05-41 (04).pdf.

-(2007): "Inequality and poverty in Latin America: a long-run exploration", en HATTON, T. S.; O'ROURKE, K. H. \& TAYLOR, A. M. (ed.), “New comparative economic history", Cambridge, MIT Press, pp. 291-315.

ROBINSON, James (2001): “Where Does Inequality Come From? Ideas and Implications for Latin America", OECD Development Center, Working Paper n. ${ }^{\circ} 188$. 
RODRÍGUEZ WEBER, Javier (2009): “Los tiempos de la desigualdad. La distribución del ingreso en Chile, entre la larga duración, la globalización y la expansión de la frontera, 1860-1930", Tesis de Maestría en Historia económica, Programa de Historia económica y social, Universidad de la República, Montevideo.

RYBCZYNSKI, T. M. (1955): "Factor endowment and relative commodity prices", en Economica, New Series, vol. 22, n. ${ }^{\circ} 88$, November, pp. 336-341.

SALAZAR, Gabriel (1985): Labradores, peones y proletarios, Ed. Sur, Santiago de Chile.

SALAZAR, Gabriel \& PINTO, Julio (2002): Historia contemporánea de Chile III. La economía: mercados, empresarios y trabajadores, Santiago, Lom.

SAMUELSON, Paul (1948): "International Trade and the Equalisation of Factor Prices", en The Economic Journal, vol. 58, n. ${ }^{\circ} 230$ (junio), pp. 163-184.

SHANAHAN, Martin \& WILSON, John (2007): "Measuring inequality trends in colonial Australia using factor-price ratios: the importance of boundaries", en Australian Economic History Review, vol. 47, n. ${ }^{\circ}$ 1, pp. 6-21.

SOCIEDAD DE FOMENTO FABRIL (1895): Censo Industrial de 1895, publicado en "Boletín de la Estadística Industrial de la república de Chile, 1894-1895", n. ${ }^{\text {os }} 1$ a 20, Santiago de Chile.

SOTO CÁRDENAS, Alejandro (1998): Influencia británica en el salitre. Origen, naturaleza y decadencia, Santiago, Universidad de Santiago.

STOLPER, Wolfgang F. \& SAMUELSON, Paul A. (1941): "Protection and real wages", en The Review of Economic Studies, vol. 9, n. ${ }^{\circ}$ 1, noviembre, pp. 58-73.

TORNERO, Ricardo (1872): Chile Ilustrado, Valparaíso, Librerías i Ajencias del Mercurio.

WAGNER, Gert (1992): “Trabajo, producción y crecimiento, la economía chilena 1860-1930", Santiago de Chile, Instituto de Economía - Pontificia Universidad Católica Documento de Trabajo n. ${ }^{\circ} 150$.

WAGNER, Gert; JOFRÉ, José \& LÜDERS, Rolf (2000): “Economía chilena 1810-1995. Cuentas Fiscales", Santiago de Chile, Instituto de Economía - Pontificia Universidad Católica Documento de Trabajo n. ${ }^{\circ} 188$.

WILLIAMSON, Jeffrey (1999): "Real wages, inequality and globalization in Latin America", en Revista de Historia Económica, Año XVII, 1999, n. ${ }^{\circ}$ especial, pp. 101142.

-(2002): "Land, Labor and Globalization in the Pre-industrial Third World", en The Journal of Economic History, vol. 62, n. ${ }^{\circ}$ 1, marzo, pp. 55-85.

-(2009): "History without evidence. Latin American inequality since 1491", NBER Working Paper n. ${ }^{\circ} 14766$. 